\title{
Catalytic Enantioselective Diarylation of Alkenes
}

\author{
Wei You and M. Kevin Brown* \\ Department of Chemistry, Indiana University \\ 800 E. Kirkwood Ave, Bloomington, IN 47405
}

\section{SUPPORTING INFORMATION: PART A}

General. Infrared (IR) spectra were recorded on an Avatar 360-FT IR E.S.P. on a $\mathrm{NaCl}$ salt plate, $v_{\max }$ in $\mathrm{cm}^{-1}$. Bands are characterized as broad (br), strong (s), medium (m), and weak (w). ${ }^{1} \mathrm{H}$ NMR spectra were recorded at room temperature on a Varian I400 (400 MHz), Varian VXR400 (400 MHz), and Varian I600 (600 MHz) spectrometer. Chemical shifts are reported in ppm from tetramethylsilane with the solvent resonance as the internal standard $\left(\mathrm{CDCl}_{3}: \delta 7.26 \mathrm{ppm}\right)$. Data is reported as follows: chemical shift, multiplicity $(\mathrm{s}=$ singlet, $\mathrm{d}=$ doublet, $\mathrm{t}=$ triplet, $\mathrm{q}=$ quartet, $\mathrm{br}=$ broad, $\mathrm{m}=$ multiplet $)$, coupling constants $(\mathrm{Hz})$, and integration. ${ }^{13} \mathrm{C}$ NMR spectra were recorded on a Varian I400 $(100 \mathrm{MHz})$ and Varian VXR400 (100 MHz) spectrometer with complete proton decoupling. Chemical shifts are reported in ppm from tetramethylsilane with the solvent resonance as the internal standard $\left(\mathrm{CDCl}_{3}: \delta 77.16 \mathrm{ppm}\right) .{ }^{31} \mathrm{P}$ NMR spectra were recorded on a Varian I400 (170 MHz) spectrometer with complete proton decoupling. Chemical shifts are reported in ppm from $85 \%$ phosphoric acid as the external standard $\left(\mathrm{H}_{3} \mathrm{PO}_{4}: \delta 0.0 \mathrm{ppm}\right)$. High-resolution mass spectrometry was performed on a Thermo Electron Corporation MAT 95XP-Trap (GC/MS). Melting points were obtained on a Thomas Hoover capillary melting point apparatus without correction. Optical rotation data were obtained on Perkin Elmer Model 343 polarimeter and are reported in terms of degree of rotation of plane-polarized light. Microwave reactions were carried out using a Biotage Initiator 60 EXP Microwave system.

Unless otherwise noted, all reactions have been carried out with distilled and degassed solvents under an atmosphere of dry $\mathrm{N}_{2}$ in oven- $\left(135{ }^{\circ} \mathrm{C}\right)$ and flame-dried glassware with standard vacuum-line techniques. Diethyl ether, tetrahydrofuran, and dichloromethane were purified under a positive pressure of dry argon by passage through two columns of activated alumina. Toluene was purified under a positive pressure of dry argon by passage through columns of activated alumina and Q5 (Grubbs apparatus). All work-up and purification procedures were carried out with reagent grade solvents (purchased from Sigma-Aldrich) in air. Standard column chromatography techniques using ZEOprep 60/40-63 $\mu \mathrm{m}$ silica gel were used for purification. 
Reagents and Catalysts:

$(\boldsymbol{R}, \boldsymbol{R})$-BenzP was purchased from Strem and used as received.

1,2-Bis(bis(3,5-dimethylphenyl)phosphanyl)benzene (dppBz-Me) was prepared in accordance with literature procedure. ${ }^{1}$

1-Bromoisoquinoline was purchased from Sigma Aldrich and used as received.

2-Bromo-3-methylpyridine was purchased from Matrix and used as received.

2-Bromo-5-methylpyridine was purchased from Sigma Aldrich and used as received.

2-Bromopyridine was purchased from Sigma Aldrich and purified through silica plug prior to use.

n-Butyl lithium (2.5 M in hexane) was purchased from Sigma Aldrich and was titrated before use.

t-Butyl lithium (1.7 M in pentane) was purchased from Sigma Aldrich and was titrated before use.

$t$-Butyl(2-iodophenoxy)dimethylsilane was was prepared in accordance with literature procedure. $^{2}$

$t$-Butyl(4-iodophenoxy)dimethylsilane was was prepared in accordance with literature procedure. $^{2}$

1-Chloro-4-iodobenzene was purchased from Combi-Blocks and used as received.

CuBr (99.999\% metals basis) was purchased from Sigma Aldrich and used as received.

CuBr-dppBz-Me, CuBr- $(\boldsymbol{R}, \boldsymbol{R})$-BenzP, and $\mathrm{CuCl}-(\boldsymbol{R}, \boldsymbol{R})-\mathrm{BenzP}$ were prepared in accordance with literature procedure. ${ }^{3}$

1,8-Diazabicycloundec-7-ene (DBU) was purchased from TCI and used as received.

Ferrocenecarboxylic acid was purchased from Alfa Aesar and used as received.

4-Iodoanisole was purchased from Oakwood and purified through silica plug prior to use.

Iodobenzene was purchased from Alfa Aesar and purified through silica plug prior to use.

2-Iodo-1,3-dimethylbenzene was purchased from Oakwood and purified through silica plug prior to use.

4-Iodopyridine was purchased from Oakwood and used as received.

2-Iodotoluene was purchased from Oakwood and purified through silica plug prior to use.

2-Isopropoxy-4,4,5,5-tetramethyl-1,3,2-dioxaborolane was purchased from Matrix and used as received.

$\mathbf{M o}(\mathbf{C O})_{6}$ was purchased from Strem and used as received.

Pinacol was purchased from Alfa Aesar and used as received.

Piperidine was purchased from Fisher and used as received.

$(\boldsymbol{R}, \boldsymbol{R})$-QuinoxP and $(\boldsymbol{S}, \boldsymbol{S})$-QuinoxP were purchased from Strem and used as received.

Sodium $\boldsymbol{t}$-butoxide was purchased from Strem and used as received. 
Tetrabutylammonium fluoride (1.0 M in THF) was purchased from Sigma Aldrich and used as received.

4,4,5,5-Tetramethyl-2-(2-((2-methylallyl)oxy)phenyl)-1,3,2-dioxaborolane

(1) was

prepared in accordance with literature procedure. ${ }^{4}$

\section{- Preparation of aryl boronic esters:}

Please note: in ${ }^{13} \mathrm{C}$ NMR spectra, signals of carbon that directly connect to boron were not detected because of quadrupolar relaxation.

\section{2-(4-Methoxy-2-((2-methylallyl)oxy)phenyl)-4,4,5,5-tetramethyl-1,3,2-dioxaborolane} (S1)

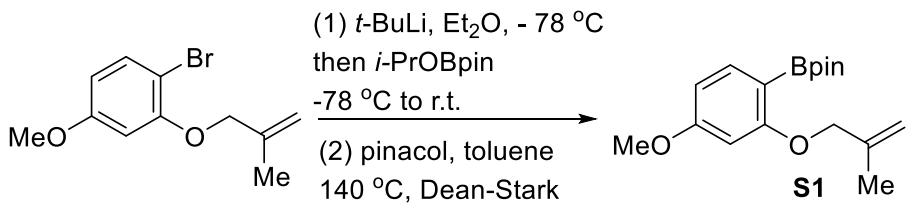

A flask containing 1-bromo-4-methoxy-2-((2-methylallyl)oxy)benzene ${ }^{5}$ (2.0 mmol, 1.0 equiv) was left under vacuum for $10 \mathrm{~min}$, then refilled with $\mathrm{N}_{2}$. This process was repeated for three times, followed by addition of $\mathrm{Et}_{2} \mathrm{O}(4.0 \mathrm{~mL})$. After cooling down to $78{ }^{\circ} \mathrm{C}$, $t$-butyl lithium (4.0 mmol, 2.0 equiv) was added dropwise over $5 \mathrm{~min}$. The mixture was allowed to stir at $-78{ }^{\circ} \mathrm{C}$ for $30 \mathrm{~min}$, followed by adding $i$-PrOBpin $(3.0$ mmol, 1.5 equiv) in one portion directly to the reaction mixture. The reaction was stirred at $-78{ }^{\circ} \mathrm{C}$ for 2 hours, then warmed up to room temperature by removing the cold bath. The mixture was stirred at room temperature for an additional hour before it was quenched with $\mathrm{H}_{2} \mathrm{O}(5 \mathrm{~mL})$ and extracted with $\mathrm{Et}_{2} \mathrm{O}(3 \times 5 \mathrm{~mL})$. The combined organic layers were dried over $\mathrm{MgSO}_{4}$, filtered, and concentrated. The residue was purified by silica gel column chromatography to obtain a mixture of S1 and the corresponding boronic acid (5:1). To the mixture was added pinacol (1.1 equiv according to $\operatorname{ArB}(\mathrm{OH})_{2}$ in the mixture) and toluene $(0.1 \mathrm{M})$. A Dean-Stark head (charged with $4 \AA$ molecule sieve) was fitted, and the reaction mixture was heated under reflux for $1 \mathrm{~h}$ (bath temperature $140{ }^{\circ} \mathrm{C}$ ). The mixture was allowed to cool to room temperature and toluene was removed under reduced pressure $(1 \mathrm{mmHg})$. The mixture was redissolved in hexane $(10 \mathrm{~mL})$ and washed with $\mathrm{H}_{2} \mathrm{O}(3 \times 5 \mathrm{~mL})$. The combined aqueous layers were extracted with hexane $(2 \times 10 \mathrm{~mL})$. Then the combined organic layers were dried over $\mathrm{MgSO}_{4}$, filtered, and concentrated to afford the title compound (S1) as a white solid without further purification (60\% yield over 2 steps): IR (neat): 3078 (w), 2977 (s), 2933 (m), 2839 (m), 1603 (s), 1572 (s), 1079 (s), 963 (m), 861 (s); ${ }^{1}$ H NMR (400 MHz, CDCl $\mathbf{~}_{3}$ ): $\delta$ $7.66(\mathrm{~d}, J=8.2 \mathrm{~Hz}, 1 \mathrm{H}), 6.48(\mathrm{dd}, J=8.2,2.0 \mathrm{~Hz}, 1 \mathrm{H}), 6.40(\mathrm{~d}, J=2.0 \mathrm{~Hz}, 1 \mathrm{H}), 5.38(\mathrm{~s}$, 1H), 4.99 (s, 1H), 4.40 (s, 2H), 3.78 (s, 3H), 1.86 (s, 3H), 1.33 (s, 12H); ${ }^{13}$ C NMR (100 MHz, $\left.\mathbf{C D C l}_{3}\right): \delta 164.9,163.6,140.7,138.2,111.7,104.6,98.9,82.9,71.3,55.1,24.9$, 19.4; HRMS (EI+): Calcd for $\mathrm{C}_{17} \mathrm{H}_{25} \mathrm{BO}_{4}\left(\mathrm{M}^{+}\right)$: 304.1840, Found 304.1834; m.p. 33-35 ${ }^{\circ} \mathrm{C}$. 


\section{2-(4-Chloro-2-((2-methylallyl)oxy)phenyl)-4,4,5,5-tetramethyl-1,3,2-dioxaborolane} (21)

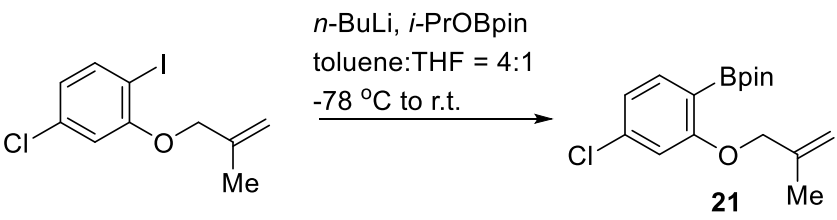

A flask containing iodo-2-((2-methylallyl)oxy)benzene ${ }^{6}(2.0 \mathrm{mmol}, 1.0$ equiv) was left under vacuum for $10 \mathrm{~min}$, then refilled with $\mathrm{N}_{2}$. This process was repeated for three times, followed by addition of $i$-PrOBpin $(2.4 \mathrm{mmol}, 1.2$ equiv) and toluene-THF $(4: 1 \mathrm{v} / \mathrm{v}$, $8.0 \mathrm{~mL}$ ). After cooling down to $-78^{\circ} \mathrm{C}, n$-butyl lithium ( $2.4 \mathrm{mmol}, 1.2$ equiv) was added dropwise over $40 \mathrm{~min} .{ }^{7}$ The mixture was allowed to stir at $-78{ }^{\circ} \mathrm{C}$ for an additional hour, and then the reaction was warmed up to room temperature over 4 hours by evaporating the cold bath slowly. The mixture was stirred at room temperature for 12 hours before it was quenched with $\mathrm{H}_{2} \mathrm{O}(5 \mathrm{~mL})$ and extracted with $\mathrm{Et}_{2} \mathrm{O}(3 \times 10 \mathrm{~mL})$. The combined organic layers were dried over $\mathrm{MgSO}_{4}$, filtered, and concentrated. The mixture was then redissolved in hexane $(5 \mathrm{~mL})$ and washed with $\mathrm{H}_{2} \mathrm{O}(3 \times 5 \mathrm{~mL})$. The combined aqueous layers were extracted with hexane $(2 \times 5 \mathrm{~mL})$. Then the combined organic layers were dried over $\mathrm{MgSO}_{4}$, filtered, and concentrated to afford the title compound (21) as a light yellow oil without further purification (72\% yield): IR (neat): 3078 (w), 2978 (s), 2932 (m), 1592 (s), 1487 (s), 1244 (s), 1019 (m), 905 (s), 743 (m), 652 (s); ${ }^{1}$ H NMR (400 MHz, $\left.\mathbf{C D C l}_{3}\right): \delta 7.61(\mathrm{~d}, J=7.9 \mathrm{~Hz}, 1 \mathrm{H}), 6.93(\mathrm{dd}, J=7.9,1.7 \mathrm{~Hz}, 1 \mathrm{H}), 6.83(\mathrm{~d}, J=1.7$ $\mathrm{Hz}, 1 \mathrm{H}), 5.35(\mathrm{~s}, 1 \mathrm{H}), 4.99(\mathrm{~s}, 1 \mathrm{H}), 4.40(\mathrm{~s}, 2 \mathrm{H}), 1.85$ (s, 3H), $1.34(\mathrm{~s}, 12 \mathrm{H}) ;{ }^{13} \mathbf{C}$ NMR $\left(100 \mathrm{MHz}, \mathbf{C D C l}_{3}\right): \delta 164.0,140.4,138.2,137.8,120.6,112.2,112.2,83.7,71.7,25.0$, 19.5; HRMS (EI+): Calcd for $\mathrm{C}_{16} \mathrm{H}_{22} \mathrm{BClO}_{3}\left(\mathrm{M}^{+}\right): 308.1345$, Found 308.1346 .

\section{4,4,5,5-Tetramethyl-2-(2-((5-methyl-2-methylenehex-5-en-1-yl)oxy)phenyl)-1,3,2- dioxaborolane (S2)}

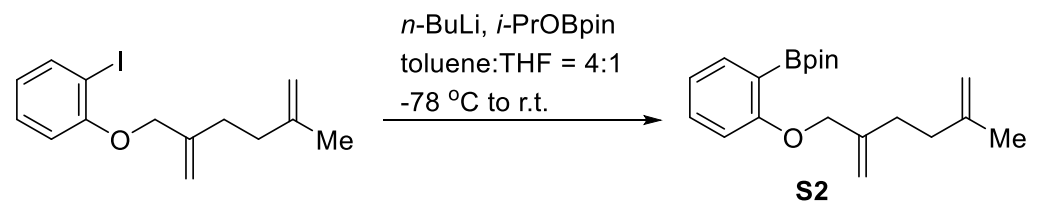

A flask containing 1-iodo-2-((5-methyl-2-methylenehex-5-en-1-yl)oxy)benzene ${ }^{8}$ (1.0 mmol, 1.0 equiv) was left under vacuum for $10 \mathrm{~min}$, then refilled with $\mathrm{N}_{2}$. This process was repeated for three times, followed by addition of $i$-PrOBpin $(1.2 \mathrm{mmol}, 1.2$ equiv) and toluene-THF $(4: 1 \mathrm{v} / \mathrm{v}, 4.0 \mathrm{~mL})$. After cooling down to $-78{ }^{\circ} \mathrm{C}, n$-butyl lithium $(1.2$ mmol, 1.2 equiv) was added dropwise over $20 \mathrm{~min}$. The mixture was allowed to stir at $78{ }^{\circ} \mathrm{C}$ for an additional hour, and then the reaction was warmed up to room temperature over 4 hours by evaporating the cold bath slowly. The mixture was stirred at room temperature for 12 hours before it was quenched with $\mathrm{H}_{2} \mathrm{O}(5 \mathrm{~mL})$ and extracted with 
$\mathrm{Et}_{2} \mathrm{O}(3 \times 10 \mathrm{~mL})$. The combined organic layers were dried over $\mathrm{MgSO}_{4}$, filtered, and concentrated. The residue was purified by silica gel column chromatography (hexane : $\left.\mathrm{Et}_{2} \mathrm{O}=10: 1\right)$ to obtain the title compound (S2) as a light yellow oil (50\% yield): IR (neat): $3076(\mathrm{w}), 2978(\mathrm{~m}), 2931$ (w), 1600 (m), 1444 (s), 1364 (s), 1315 (m), 1145 (m), 756 (m), 658 (w); ${ }^{1} \mathbf{H}$ NMR (400 MHz, $\left.\mathbf{C D C l}_{3}\right): \delta 7.74$ (d, $\left.J=7.2 \mathrm{~Hz}, 1 \mathrm{H}\right), 7.40$ (app. t, $J=7.7 \mathrm{~Hz}, 1 \mathrm{H}$ ), 6.97 (app. t, $J=7.3 \mathrm{~Hz}, 1 \mathrm{H}), 6.87$ (d, $J=8.3 \mathrm{~Hz}, 1 \mathrm{H}), 5.43$ (s, 1H), 5.05 $(\mathrm{s}, 1 \mathrm{H}), 4.77(\mathrm{~s}, 2 \mathrm{H}), 4.50(\mathrm{~s}, 2 \mathrm{H}), 2.44-2.36(\mathrm{~m}, 2 \mathrm{H}), 2.30-2.22(\mathrm{~m}, 2 \mathrm{H}), 1.79(\mathrm{~s}, 3 \mathrm{H})$, 1.37 (s, 12H); ${ }^{13} \mathbf{C}$ NMR (100 MHz, $\left.\mathbf{C D C l}_{3}\right): \delta 163.3,145.4,144.7,136.9,132.5,120.3$, 111.4, 111.3, 110.2, 83.4, 70.6, 35.8, 31.2, 24.9, 22.6; HRMS (EI+): Calcd for $\mathrm{C}_{20} \mathrm{H}_{29} \mathrm{BO}_{3}\left(\mathrm{M}^{+}\right): 328.2204$, Found 328.2216.

\section{4,4,5,5-Tetramethyl-2-(2-((2-methylenehexyl)oxy)phenyl)-1,3,2-dioxaborolane (S3)}

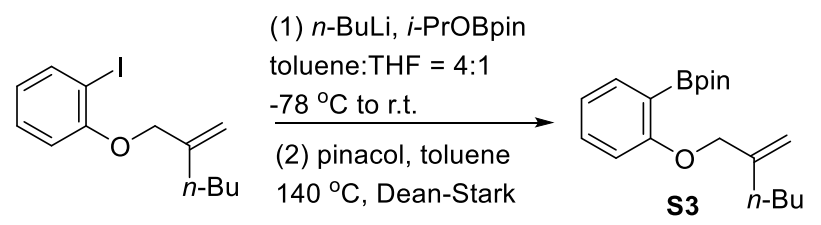

A flask containing 1-iodo-2-((5-methyl-2-methylenehex-5-en-1-yl)oxy)benzene ${ }^{9}$ (1.0 mmol, 1.0 equiv) was left under vacuum for $10 \mathrm{~min}$, the refilled with $\mathrm{N}_{2}$. This process was repeated for three times, followed by addition of $i$-PrOBpin (1.2 mmol, 1.2 equiv) and toluene-THF $(4: 1 \mathrm{v} / \mathrm{v}, 4.0 \mathrm{~mL})$. After cooling down to $-78{ }^{\circ} \mathrm{C}, n$-butyl lithium $(1.2$ mmol, 1.2 equiv) was added dropwise over $20 \mathrm{~min}$. The mixture was allowed to stir at $78{ }^{\circ} \mathrm{C}$ for an additional hour, and then the reaction was warmed up to room temperature over 4 hours by evaporating the cold bath slowly. The mixture was stirred at room temperature for 12 hours before it was quenched with $\mathrm{H}_{2} \mathrm{O}(5 \mathrm{~mL})$ and extracted with $\mathrm{Et}_{2} \mathrm{O}(3 \times 10 \mathrm{~mL})$. The combined organic layers were dried over $\mathrm{MgSO}_{4}$, filtered, and concentrated. The residue was purified by silica gel column chromatography to obtain a mixture of S3 and the corresponding boronic acid (2.5:1). To the mixture was added pinacol (1.1 equiv according to $\mathrm{ArB}(\mathrm{OH})_{2}$ in the mixture) and toluene $(0.1 \mathrm{M})$. A DeanStark head (charged with $4 \AA$ molecule sieve) was fitted, and the reaction mixture was heated under reflux for $1 \mathrm{~h}$ (bath temperature $140{ }^{\circ} \mathrm{C}$ ). The mixture was allowed to cool to room temperature and toluene was removed under reduced pressure $(1 \mathrm{mmHg})$. The mixture was redissolved in hexane $(5 \mathrm{~mL})$ and washed with $\mathrm{H}_{2} \mathrm{O}(3 \times 5 \mathrm{~mL})$. The combined aqueous layers were extracted with hexane $(2 \times 5 \mathrm{~mL})$. Then the combined organic layers were dried over $\mathrm{MgSO}_{4}$, filtered, and concentrated to afford the title compound (S3) as a colorless oil without further purification (85\% yield over 2 steps): IR (neat): 3081 (w), 2977 (m), 2930 (m), 1600 (m), 1443 (s), 1355 (s), 1146 (m), 904 (w), $758(\mathrm{~m}) ;{ }^{1} \mathbf{H}$ NMR (400 MHz, $\left.\mathbf{C D C l}_{3}\right): \delta 7.75$ (dd, $\left.J=7.2,1.5 \mathrm{~Hz}, 1 \mathrm{H}\right), 7.40$ (app. td, $J$ $=7.8,1.5 \mathrm{~Hz}, 1 \mathrm{H}$ ), 6.97 (app. t, $J=7.3 \mathrm{~Hz}, 1 \mathrm{H}), 6.87$ (d, $J=8.3 \mathrm{~Hz}, 1 \mathrm{H}), 5.45(\mathrm{~s}, 1 \mathrm{H})$, $5.03(\mathrm{~s}, 1 \mathrm{H}), 4.48(\mathrm{~s}, 2 \mathrm{H}), 2.23(\mathrm{t}, J=7.6 \mathrm{~Hz}, 2 \mathrm{H}), 1.58-1.48(\mathrm{~m}, 2 \mathrm{H}), 1.47-1.35(\mathrm{~m}, 2 \mathrm{H})$, 1.38 (s, 12H), $0.97(\mathrm{t}, J=7.3 \mathrm{~Hz}, 3 \mathrm{H}) ;{ }^{13} \mathbf{C}$ NMR (100 MHz, $\left.\mathbf{C D C l}_{3}\right): \delta 163.3,144.9$, $136.8,132.4,120.2,111.4,110.7,83.2,70.5,32.8,29.8,24.9,22.5,14.0$; HRMS (EI+): Calcd for $\mathrm{C}_{19} \mathrm{H}_{29} \mathrm{BO}_{3}\left(\mathrm{M}^{+}\right): 316.2204$, Found 316.2200. 
4,4,5,5-Tetramethyl-2-(2-(3-methyl-2-methylenebutoxy)phenyl)-1,3,2-dioxaborolane (S4)

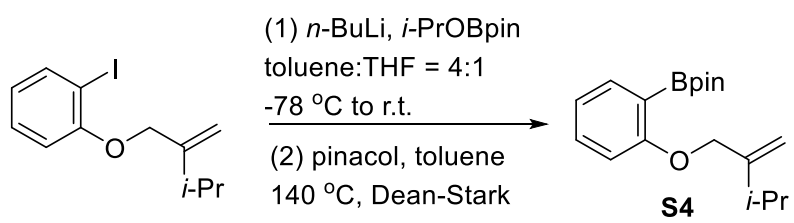

A flask containg 1-iodo-2-((5-methyl-2-methylenehex-5-en-1-yl)oxy)benzene ${ }^{10}$ (1.0 mmol, 1.0 equiv) was left under vacuum for $10 \mathrm{~min}$, then refilled with $\mathrm{N}_{2}$. This process was repeated for three times, followed by addition of $i$-PrOBpin (1.2 mmol, 1.2 equiv) and toluene-THF $(4: 1 \mathrm{v} / \mathrm{v}, 4.0 \mathrm{~mL})$. After cooling down to $-78{ }^{\circ} \mathrm{C}, n$-butyl lithium $(1.2$ mmol, 1.2 equiv) was added dropwise over $20 \mathrm{~min}$. The mixture was allowed to stir at $78{ }^{\circ} \mathrm{C}$ for an additional hour, and then the reaction was warmed up to room temperature over 4 hours by evaporating the cold bath slowly. The mixture was stirred at room temperature for 12 hours before it was quenched with $\mathrm{H}_{2} \mathrm{O}(5 \mathrm{~mL})$ and extracted with $\mathrm{Et}_{2} \mathrm{O}(3 \times 10 \mathrm{~mL})$. The combined organic layers were dried over $\mathrm{MgSO}_{4}$, filtered, and concentrated. The residue was purified by silica gel column chromatography to obtain a mixture of $\mathbf{S 4}$ and the corresponding boronic acid (2:1). To the mixture was added pinacol (1.1 equiv according to $\mathrm{ArB}(\mathrm{OH})_{2}$ in the mixture) and toluene $(0.1 \mathrm{M})$. A DeanStark head (charged with $4 \AA$ molecule sieve) was fitted, and the reaction mixture was heated under reflux for $1 \mathrm{~h}$ (bath temperature $140{ }^{\circ} \mathrm{C}$ ). The mixture was allowed to cool to room temperature and toluene was removed under reduced pressure $(1 \mathrm{mmHg})$. The mixture was redissolved in hexane $(5 \mathrm{~mL})$ and washed with $\mathrm{H}_{2} \mathrm{O}(3 \times 5 \mathrm{~mL})$. The combined aqueous layers were extracted with hexane $(2 \times 5 \mathrm{~mL})$. Then the combined organic layers were dried over $\mathrm{MgSO}_{4}$, filtered, and concentrated to afford the title compound (S4) as a colorless oil without further purification ( $88 \%$ yield over 2 steps): IR (neat): 3073 (w), 2976 (s), 2871 (m), 1600 (s), 1489 (m), 1443 (s), 1355 (s), 1146 (m), 1071 (m), 907 (w), 759 (m); ${ }^{1} \mathbf{H}$ NMR (400 MHz, $\left.\mathbf{C D C l}_{3}\right): \delta 7.74(\mathrm{dd}, J=7.3,1.7 \mathrm{~Hz}$, 1H), 7.40 (app. td, $J=8.4,1.8 \mathrm{~Hz}, 1 \mathrm{H}), 6.96$ (app. t, $J=7.3 \mathrm{~Hz}, 1 \mathrm{H}), 6.88$ (d, $J=8.3 \mathrm{~Hz}$, $1 \mathrm{H}), 5.46(\mathrm{~d}, J=1.2 \mathrm{~Hz}, 1 \mathrm{H}), 5.05(\mathrm{~s}, 1 \mathrm{H}), 4.54$ (s, 2H), 2.56 (hept, $J=6.9 \mathrm{~Hz}, 1 \mathrm{H}), 1.37$ $(\mathrm{s}, 12 \mathrm{H}), 1.16(\mathrm{~d}, J=6.9 \mathrm{~Hz}, 6 \mathrm{H}) ;{ }^{13} \mathbf{C}$ NMR (100 MHz, $\left.\mathbf{C D C l}_{3}\right): \delta 163.3,150.7,136.8$, 132.5, 120.2, 111.4, 108.9, 83.3, 69.8, 30.8, 24.9, 21.7; HRMS (EI+): Calcd for $\mathrm{C}_{18} \mathrm{H}_{27} \mathrm{BO}_{3}\left(\mathrm{M}^{+}\right):$302.2048, Found 302.2045.

\section{$N$-Benzyl- $N$-(2-methylallyl)-2-(4,4,5,5-tetramethyl-1,3,2-dioxaborolan-2-yl)aniline} (S5)

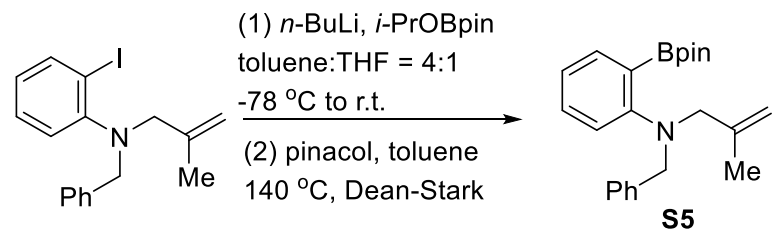


A flask containing $N$-benzyl-2-iodo- $N$-(2-methylallyl)aniline ${ }^{11}$ (1.0 mmol, 1.0 equiv) was left under vacuum for $10 \mathrm{~min}$, then refilled with $\mathrm{N}_{2}$. This process was repeated for three times, followed by addition of $i$-PrOBpin ( $1.2 \mathrm{mmol}, 1.2$ equiv) and toluene-THF $(4: 1 \mathrm{v} / \mathrm{v}$, $4.0 \mathrm{~mL})$. After cooling down to $-78^{\circ} \mathrm{C}, n$-butyl lithium $(1.2 \mathrm{mmol}, 1.2$ equiv $)$ was added dropwise over $20 \mathrm{~min}$. The mixture was allowed to stir at $-78{ }^{\circ} \mathrm{C}$ for an additional hour, and then the reaction was warmed up to room temperature over 4 hours by evaporating the cold bath slowly. The mixture was stirred at room temperature for 12 hours before it was quenched with $\mathrm{H}_{2} \mathrm{O}(5 \mathrm{~mL})$ and extracted with $\mathrm{Et}_{2} \mathrm{O}(3 \times 10 \mathrm{~mL})$. The combined organic layers were dried over $\mathrm{MgSO}_{4}$, filtered, and concentrated. The residue was purified by silica gel column chromatography to obtain a mixture of $\mathbf{S 5}$ and the corresponding boronic acid (5:1). To the mixture was added pinacol (1.1 equiv according to $\operatorname{ArB}(\mathrm{OH})_{2}$ in the mixture) and toluene $(0.1 \mathrm{M})$. A Dean-Stark head (charged with $4 \AA$ molecule sieve) was fitted, and the reaction mixture was heated under reflux for $1 \mathrm{~h}$ (bath temperature $140{ }^{\circ} \mathrm{C}$ ). The mixture was allowed to cool to room temperature and toluene was removed under reduced pressure $(1 \mathrm{mmHg})$. The mixture was redissolved in hexane $(5 \mathrm{~mL})$ and washed with $\mathrm{H}_{2} \mathrm{O}(3 \times 5 \mathrm{~mL})$. The combined aqueous layers were extracted with hexane $(2 \times 5 \mathrm{~mL})$. Then the combined organic layers were dried over $\mathrm{MgSO}_{4}$, filtered, and concentrated to afford the title compound (S5) as a yellow oil without further purification (52\% yield over 2 steps): IR (neat): 3063 (w), 3026 (w), 2977 (s), 2929 (m), 1593 (s), 1486 (s), 1371 (s), 1213 (m), 1145 (s), 897 (m), 663 (m); ${ }^{1}$ H NMR (400 MHz, $\left.\mathbf{C D C l}_{3}\right): \delta 7.85(\mathrm{dd}, J=7.3,1.5 \mathrm{~Hz}, 1 \mathrm{H}), 7.44-7.28(\mathrm{~m}, 6 \mathrm{H}), 7.02$ (app. t, $J=7.2 \mathrm{~Hz}$, $1 \mathrm{H}), 6.96(\mathrm{~d}, J=8.2 \mathrm{~Hz}, 1 \mathrm{H}), 4.98(\mathrm{~s}, 2 \mathrm{H}), 4.46(\mathrm{~s}, 2 \mathrm{H}), 3.72(\mathrm{~s}, 2 \mathrm{H}), 1.83(\mathrm{~s}, 3 \mathrm{H}), 1.45$ $(\mathrm{s}, 12 \mathrm{H}) ;{ }^{13} \mathbf{C}$ NMR (100 MHz, $\mathbf{C D C l}$ ): $\delta$ 157.5, 142.9, 138.6, 136.8, 131.2, 128.7, 127.9, 126.8, 120.4, 119.8, 113.0, 83.3, 58.1, 25.0, 20.8; HRMS (EI+): Calcd for $\mathrm{C}_{23} \mathrm{H}_{30} \mathrm{BNO}_{2}\left(\mathrm{M}^{+}\right):$363.2364, Found 363.2355.

\section{a General Procedure for Racemic Diarylation of Alkenes:}

In an $\mathrm{N}_{2}$ filled glovebox, to a $\sim 10 \mathrm{~mL}$ screw-capped vial $(13 \times 100 \mathrm{~mm}$ ) was added $\mathrm{CuBr}-$ dppBz-Me (0.005 mmol, $5 \mathrm{~mol} \%), \mathrm{NaO} t$-Bu (0.1 mmol, 1.0 equiv), ArBpin ( $0.1 \mathrm{mmol}$, 1.0 equiv), aryl iodide ( $0.3 \mathrm{mmol}, 3.0$ equiv) and toluene $(3.0 \mathrm{~mL})$. The vial was sealed with a Teflon lined screw cap and then removed from the glovebox. The mixture was allowed to stir at $120{ }^{\circ} \mathrm{C}$ for $12 \mathrm{~h}$. After cooling down to room temperature, the reaction was quenched upon addition of $\mathrm{H}_{2} \mathrm{O}(2 \mathrm{~mL})$ and extracted with EtOAc $(3 \times 2 \mathrm{~mL})$. The combined organic layers were dried over $\mathrm{MgSO}_{4}$, filtered, and concentrated. The residue was purified by silica gel column chromatography (hexane: dichloromethane) to obtain the desired product.

\section{ש General Procedure for Enantioselective Diarylation of Alkenes:}

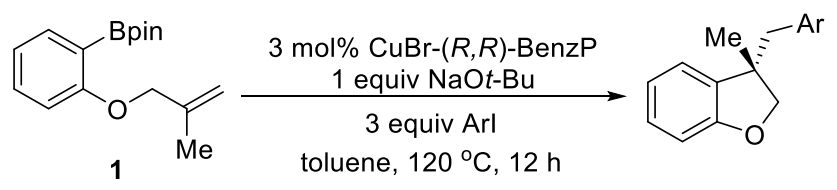


In an $\mathrm{N}_{2}$ filled glovebox, to a $\sim 10 \mathrm{~mL}$ screw-capped vial $(13 \times 100 \mathrm{~mm})$ was added $\mathrm{CuBr}-$ $(R, R)$-BenzP (0.003 mmol, $3 \mathrm{~mol} \%), \mathrm{NaOt}$-Bu (0.1 mmol, 1.0 equiv), ArBpin ( $0.1 \mathrm{mmol}$, 1.0 equiv), aryl iodide $(0.3 \mathrm{mmol}, 3.0$ equiv) and toluene $(3.0 \mathrm{~mL})$. The vial was sealed with a Teflon lined screw cap and then removed from the glovebox. The mixture was allowed to stir at $120^{\circ} \mathrm{C}$ for $12 \mathrm{~h}$. After cooling down to room temperature, the reaction was quenched upon addition of $\mathrm{H}_{2} \mathrm{O}(2 \mathrm{~mL})$ and extracted with EtOAc $(3 \times 2 \mathrm{~mL})$. The combined organic layers were dried over $\mathrm{MgSO}_{4}$, filtered, and concentrated. The residue was purified by silica gel column chromatography (hexane : dichloromethane) to obtain the desired product.

\section{- Optimization Table:}

Detailed optimization table is shown in Table S1 (compared with Table 1 in text). Yield of direct-coupling product (d.c. product) is included.

Table S1. Reaction Optimization

\begin{tabular}{|c|c|c|c|c|c|}
\hline & $\mathrm{Me}$ & 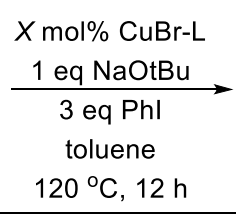 & (N) & d.c. proo & duct \\
\hline entry & $\mathrm{X} \mathrm{mol} \%$ & ligand & yield of $\mathbf{2}(\%)^{a}$ & yield of d.c. $(\%)^{a}$ & e.r. ${ }^{b}$ \\
\hline 1 & 5 & dppBz-Me & 78 & 4 & -- \\
\hline 2 & 5 & $(R, R)$-Me-DuPhos & 70 & 10 & $60: 40$ \\
\hline 3 & 5 & $(S, S)$-iPr-DuPhos & 34 & 29 & $82: 18$ \\
\hline 4 & 5 & $(R, R)$-BenzP & 76 & 7 & $97: 3$ \\
\hline 5 & 5 & $(R, R)$-QuinoxP & 69 & 6 & $92: 8$ \\
\hline 8 & 3 & $(R, R)-B e n z P$ & 77 & 7 & 98:2 \\
\hline 9 & 1 & $(R, R)$-BenzP & 36 & 9 & $97: 3$ \\
\hline $10^{c}$ & 3 & $(R, R)$-BenzP & $<5$ & n.d. & n.d. \\
\hline
\end{tabular}

${ }^{a}$ Determined by ${ }^{1} \mathrm{H}$ NMR analysis $(400 \mathrm{MHz})$ with an internal standard. ${ }^{b}$ Determined by HPLC analysis with a chiral column. ${ }^{\circ}$ Reaction at $100^{\circ} \mathrm{C}$.

\section{Characterization Data:}

(+)-(S)-3-Benzyl-3-methyl-2,3-dihydrobenzofuran (2): 2 was prepared according to general procedure above using iodobenzene and was purified by silica gel column chromatography (Hex : DCM = 15:1) to obtain 2 as colorless oil (72\% yield, 98:2 er). 7\% direct cross-coupling byproduct was observed in the crude reaction mixture. IR (neat): 3061 (w), 3028 (m), 2925 (m), 2875 (m), 1597 (m), 1480 (s), 1458 (m), 976 (m), 750 (s); ${ }^{1} \mathbf{H}$ NMR (400 MHz, CDCl$\left.)_{3}\right): \delta 7.29-7.22(\mathrm{~m}, 3 \mathrm{H}), 7.17-7.12(\mathrm{~m}, 1 \mathrm{H}), 7.03-6.99(\mathrm{~m}, 2 \mathrm{H})$, $6.95(\mathrm{~d}, J=7.3 \mathrm{~Hz}, 1 \mathrm{H}), 6.87$ (app. t, $J=7.4 \mathrm{~Hz}, 1 \mathrm{H}), 6.78(\mathrm{~d}, J=8.0 \mathrm{~Hz}, 1 \mathrm{H}), 4.51$ (d, $J$ $=8.7 \mathrm{~Hz}, 1 \mathrm{H}), 4.07(\mathrm{~d}, J=8.7 \mathrm{~Hz}, 1 \mathrm{H}), 2.92(\mathrm{~d}, J=13.3 \mathrm{~Hz}, 1 \mathrm{H}), 2.87(\mathrm{~d}, J=13.3 \mathrm{~Hz}$, 1H), 1.37 (s, 3H); ${ }^{13} \mathbf{C}$ NMR (100 MHz, $\left.\mathbf{C D C l}_{3}\right)$ : $\delta$ 159.7, 137.7, 135.0, 130.5, 128.3, 
128.1, 126.6, 123.5, 120.4, 109.8, 82.0, 46.8, 46.4, 24.7; HRMS (CI+): Calcd for $\mathrm{C}_{16} \mathrm{H}_{17} \mathrm{O}\left(\mathrm{M}^{+}+\mathrm{H}\right): 225.1274$, Found 225.1274; Optical Rotation: $[\alpha]_{\mathrm{D}}{ }^{20}+13.6(c 0.75$ in $\mathrm{CHCl}_{3}$ ) for an enantiomerically enriched sample of 97:3 er. The enantiomeric purity was established by HPLC analysis using a chiral column (Chiralpak IA column, $22{ }^{\circ} \mathrm{C}, 0.75$ $\mathrm{mL} / \mathrm{min}$, 99.8:0.2 Hexane:Isopropanol, $254 \mathrm{~nm}, \mathrm{t}_{\text {minor }}=17.187 \mathrm{~min}, \mathrm{t}_{\text {major }}=21.424 \mathrm{~min}$ ). Absolute stereochemistry was determined through analogy with S10. See supporting information part B for HPLC chromatograms.

Large scale procedure: In an $\mathrm{N}_{2}$ filled glovebox, to a $\sim 100 \mathrm{~mL}$ screw-capped vial $(45 \mathrm{x}$ $120 \mathrm{~mm}$ ) was added $\mathrm{CuBr}-(R, R)$-BenzP (0.03 mmol, $3 \mathrm{~mol} \%), \mathrm{NaO} t$-Bu (1.0 mmol, 1.0 equiv), ArBpin 1 (1.0 mmol, $274.2 \mathrm{mg}, 1.0$ equiv), iodobenzene (3.0 mmol, 3.0 equiv) and toluene $(30 \mathrm{~mL})$. The vial was sealed with a Teflon lined screw cap and then removed from the glovebox, and the mixture was allowed to stir at $120{ }^{\circ} \mathrm{C}$ for $12 \mathrm{~h}$. After cooling to room temperature, the reaction was quenched upon addition of $\mathrm{H}_{2} \mathrm{O}(10 \mathrm{~mL})$ and the mixture was extracted with EtOAc $(3 \mathrm{x} 10 \mathrm{~mL})$. The combined organic layers were washed with brine and dried over $\mathrm{MgSO}_{4}$, filtered, and concentrated. The residue was purified by silica gel column chromatography (Hex : $\mathrm{DCM}=15: 1$ ) to obtain 2 as colorless oil (156.7 mg, 70\% yield, 97:3 er). See supporting information part B for HPLC chromatograms.

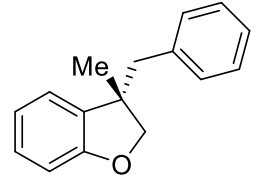

(-)-(R)-3-Benzyl-3-methyl-2,3-dihydrobenzofuran ((-)-2): (-)-2 was prepared according to general procedure above using $5 \mathrm{~mol} \% \mathrm{CuBr}, 5 \mathrm{~mol} \%(S, S)$-QuinoxP and iodobenzene and was purified by silica gel column chromatography (Hex : DCM $=15: 1$ ) to obtain (-)2 as colorless oil (61\% yield, 94:6 er). $6 \%$ direct cross-coupling byproduct was observed in the crude reaction mixture. See supporting information part B for HPLC chromatograms.

Note: The pre-mix complex of CuBr-QuinoxP is not an active catalyst for the reaction, so $\mathrm{CuBr}$ and $(S, S)$-QuinoxP have to be added separately. The equivalence of $\mathrm{CuBr}$ and ligand affects the reaction result. Excess ligand can cause diminished yield, and excess $\mathrm{CuBr}$ can cause decreased enantioselectivity.

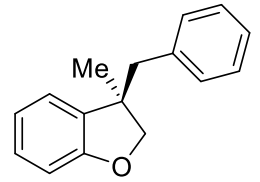

(+)-(S)-3-(4-Chlorobenzyl)-3-methyl-2,3-dihydrobenzofuran (3): 3 was prepared according to general procedure above using 1-chloro-4-iodobenzene and was purified by silica gel column chromatography (Hex : DCM $=15: 1)$ to obtain 3 as colorless oil (41\% yield, 97.5:2.5 er). 10\% direct cross-coupling byproduct was observed in the crude reaction mixture. IR (neat): 3028 (w), 2962 (m), 2925 (m), 2876 (w), 1596 (m), 1480 (s), 
1459 (m), 1016 (m), 752 (m); ${ }^{1} \mathbf{H}$ NMR (400 MHz, $\left.\mathbf{C D C l}_{3}\right): \delta 7.20$ (d, J= 7.9 Hz, 2H), 7.14 (app. t, $J=7.6 \mathrm{~Hz}, 1 \mathrm{H}), 6.94-6.83(\mathrm{~m}, 4 \mathrm{H}), 6.76(\mathrm{~d}, J=8.0 \mathrm{~Hz}, 1 \mathrm{H}), 4.45$ (d, $J=8.7$ $\mathrm{Hz}, 1 \mathrm{H}), 4.08(\mathrm{~d}, J=8.7 \mathrm{~Hz}, 1 \mathrm{H}), 2.86(\mathrm{~d}, J=13.7 \mathrm{~Hz}, 1 \mathrm{H}), 2.82(\mathrm{~d}, J=13.8 \mathrm{~Hz}, 1 \mathrm{H})$, 1.36 (s, 3H); ${ }^{13} \mathbf{C}$ NMR (100 MHz, $\left.\mathbf{C D C l}_{3}\right): \delta 159.7,136.1,134.3,132.6,131.7,128.5$, 128.2, 123.5, 120.5, 109.9, 82.0, 46.3, 46.2, 24.6; HRMS (CI+): Calcd for $\mathrm{C}_{16} \mathrm{H}_{16} \mathrm{ClO}$ $\left(\mathrm{M}^{+}+\mathrm{H}\right): 259.0884$, Found 259.0884; Optical Rotation: $[\alpha]_{\mathrm{D}}{ }^{20}+24.1\left(c 0.75\right.$ in $\left.\mathrm{CHCl}_{3}\right)$ for an enantiomerically enriched sample of 97:3 er. The enantiomeric purity was established by HPLC analysis using a chiral column (Chiralpak IA column, $22{ }^{\circ} \mathrm{C}, 0.5$ $\mathrm{mL} / \mathrm{min}$, 99:1 Hexane:Isopropanol, $254 \mathrm{~nm}, \mathrm{t}_{\text {major }}=9.528 \mathrm{~min}, \mathrm{t}_{\mathrm{minor}}=10.510 \mathrm{~min}$ ). Absolute stereochemistry was determined through analogy with S10. See supporting information part B for HPLC chromatograms.

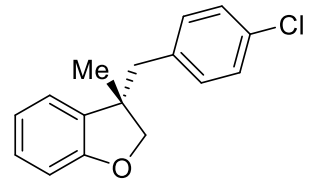

(+)-(S)-3-(4-Methoxybenzyl)-3-methyl-2,3-dihydrobenzofuran (4): 4 was prepared according to general procedure above using 4-iodoanisole and was purified by silica gel column chromatography (Hex : $\mathrm{DCM}=2: 1$ ) to obtain 4 as colorless oil (62\% yield, 98:2 er). $9 \%$ direct cross-coupling byproduct was observed in the crude reaction mixture. IR (neat): 3031 (m), 2956 (s), 2927 (s), 2835 (m), 1611 (s), 1461 (s), 1248 (s), 1151 (m), 1036 (s), 753 (s); ${ }^{1} \mathbf{H}$ NMR (400 MHz, $\mathbf{C D C l}_{3}$ ): $\delta 7.13$ (app. t, $J=7.6 \mathrm{~Hz}, 1 \mathrm{H}$ ), 6.97-6.83 (m, 4H), 6.82-6.73 (m, 3H), $4.48(\mathrm{~d}, J=8.7 \mathrm{~Hz}, 1 \mathrm{H}), 4.06(\mathrm{~d}, J=8.7 \mathrm{~Hz}, 1 \mathrm{H}), 3.79$ (s, $3 \mathrm{H}), 2.85(\mathrm{~d}, J=13.5 \mathrm{~Hz}, 1 \mathrm{H}), 2.80(\mathrm{~d}, J=13.5 \mathrm{~Hz}, 1 \mathrm{H}), 1.35(\mathrm{~s}, 3 \mathrm{H}) ;{ }^{13} \mathrm{C}$ NMR (100 MHz, $\left.\mathbf{C D C l}_{3}\right): \delta 159.7,158.4,135.0,131.4,129.8,128.3,123.5,120.4,113.5,109.8$, 82.0, 55.3, 46.5, 45.9, 24.7; HRMS (CI+): Calcd for $\mathrm{C}_{17} \mathrm{H}_{19} \mathrm{O}_{2}\left(\mathrm{M}^{+}+\mathrm{H}\right)$ : 255.1380, Found 255.1385; Optical Rotation: $[\alpha]_{\mathrm{D}}{ }^{20}+17.5$ (c 0.75 in $\left.\mathrm{CHCl}_{3}\right)$ for an enantiomerically enriched sample of 97:3 er. The enantiomeric purity was established by HPLC analysis using a chiral column (Chiralpak IA column, $22{ }^{\circ} \mathrm{C}, 0.5 \mathrm{~mL} / \mathrm{min}, 99: 1$ Hexane:Isopropanol, $254 \mathrm{~nm}, \mathrm{t}_{\text {major }}=11.008 \mathrm{~min}, \mathrm{t}_{\text {minor }}=11.900 \mathrm{~min}$ ). Absolute stereochemistry was determined through analogy with S10. See supporting information part B for HPLC chromatograms.

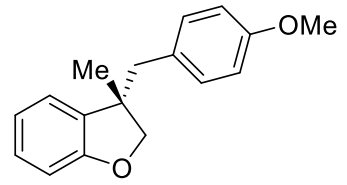

(-)-(S)-t-Butyldimethyl(2-((3-methyl-2,3-dihydrobenzofuran-3-yl)methyl)phenoxy)

silane (5): 5 was prepared according to general procedure above using $t$-butyl(2iodophenoxy)dimethylsilane and was purified by silica gel column chromatography (Hex : $\mathrm{DCM}=8: 1)$ to obtain 5 as colorless oil (61\% yield, 97:3 er). 7\% direct cross-coupling 
byproduct was observed in the crude reaction mixture. IR (neat): 3028 (w), 2957 (s), 2929 (s), 2858 (m), 1597 (m), 1490 (s), 1264 (s), 925 (s), 836 (s), 748 (s); ${ }^{\mathbf{1}}$ H NMR (400 MHz, $\left.\mathbf{C D C l}_{3}\right): \delta$ 7.16-7.02 (m, 3H), 6.88 (app. t, $\left.J=7.5 \mathrm{~Hz}, 1 \mathrm{H}\right), 6.85-6.69(\mathrm{~m}, 4 \mathrm{H})$, $4.58(\mathrm{~d}, J=8.7 \mathrm{~Hz}, 1 \mathrm{H}), 4.04(\mathrm{~d}, J=8.7 \mathrm{~Hz}, 1 \mathrm{H}), 3.06(\mathrm{~d}, J=13.2 \mathrm{~Hz}, 1 \mathrm{H}), 2.84(\mathrm{~d}, J=$ $13.2 \mathrm{~Hz}, 1 \mathrm{H}), 1.37$ (s, 3H), 1.02 (s, 9H), 0.24 (s, 6H); $\left.{ }^{13} \mathbf{C ~ N M R ~ ( 1 0 0 ~ M H z , ~ C D C l ~}\right): \delta$ 159.7, 154.3, 135.5, 132.2, 128.6, 128.2, 127.6, 123.2, 120.7, 120.4, 118.8, 109.8, 82.0, 47.1, 39.6, 26.1, 25.4, 18.5, -3.9, -4.0; HRMS (CI+): Calcd for $\mathrm{C}_{22} \mathrm{H}_{31} \mathrm{O}_{2} \mathrm{Si}\left(\mathrm{M}^{+}+\mathrm{H}\right)$ : 355.2088, Found 355.2083; Optical Rotation: $[\alpha]_{\mathrm{D}}{ }^{20}-31.2\left(c 0.75\right.$ in $\left.\mathrm{CHCl}_{3}\right)$ for an enantiomerically enriched sample of 97:3 er. Enantiopurity was determined through deprotection to phenol S6 (see below). Absolute stereochemistry was determined through analogy with S10.

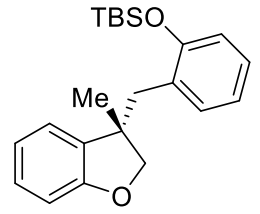

(-)-(S)-2-((3-Methyl-2,3-dihydrobenzofuran-3-yl)methyl)phenol $\quad$ (S6): $\quad$ S6 was prepared from $\mathbf{5}$ according to modified literature procedure. ${ }^{12}$ A solution of the silyl ether 5 (0.1 mmol, 1.0 equiv) in dry THF $(1 \mathrm{~mL})$ was treated with tetrabutylammonium fluoride (1.0 M solution in THF, $0.3 \mathrm{mmol}, 3.0$ equiv), and was allowed to stir at room temperature for $3 \mathrm{~h}$. The reaction was quenched with $\mathrm{H}_{2} \mathrm{O}(2 \mathrm{~mL})$ and brine $(2 \mathrm{~mL})$ and the mixture was extracted with $\mathrm{Et}_{2} \mathrm{O}(3 \times 3 \mathrm{~mL})$. The combined organic layers were dried over $\mathrm{MgSO}_{4}$, filtered, and concentrated. The residue was purified by silica gel column chromatography $\left(\mathrm{Hex}: \mathrm{Et}_{2} \mathrm{O}=1: 1\right)$ to obtain the desired product in $94 \%$ yield. IR (neat): 3406 (br s), 3032 (w), 2961 (m), 2925 (m), 1595 (s), 1479 (s), 1458 (s), 968 (m), 751 (s); ${ }^{1} \mathbf{H}$ NMR (400 MHz, $\mathbf{C D C l}_{3}$ ): $\delta 7.15$ (app. td, $\left.J=7.7,1.4 \mathrm{~Hz}, 1 \mathrm{H}\right), 7.10$ (app. td, $J=7.7$, $1.6 \mathrm{~Hz}, 1 \mathrm{H}), 7.02(\mathrm{~d}, J=7.4 \mathrm{~Hz}, 1 \mathrm{H}), 6.93-6.86(\mathrm{~m}, 2 \mathrm{H}), 6.85-6.76(\mathrm{~m}, 2 \mathrm{H}), 6.73(\mathrm{~d}, J=$ $8.0 \mathrm{~Hz}, 1 \mathrm{H}), 4.62(\mathrm{~d}, J=8.7 \mathrm{~Hz}, 1 \mathrm{H}), 4.56(\mathrm{br} \mathrm{s}, 1 \mathrm{H}), 4.10(\mathrm{~d}, J=8.7 \mathrm{~Hz}, 1 \mathrm{H}), 3.00(\mathrm{~d}, J$ $=13.6 \mathrm{~Hz}, 1 \mathrm{H}), 2.89(\mathrm{~d}, J=13.6 \mathrm{~Hz}, 1 \mathrm{H}), 1.41(\mathrm{~s}, 3 \mathrm{H}) ;{ }^{13} \mathbf{C}$ NMR $\left(\mathbf{1 0 0} \mathbf{M H z}, \mathbf{C D C l}_{3}\right): \delta$ 159.6, 154.2, 135.1, 132.7, 128.5, 128.1, 124.3, 123.2, 120.7, 120.7, 115.9, 110.0, 82.5, 46.9, 40.0, 24.5; HRMS (CI+): Calcd for $\mathrm{C}_{16} \mathrm{H}_{16} \mathrm{O}_{2}\left(\mathrm{M}^{+}\right)$: 240.1145, Found 240.1142; Optical Rotation: $[\alpha]_{\mathrm{D}}{ }^{20}-2.8\left(c 0.75\right.$ in $\left.\mathrm{CHCl}_{3}\right)$ for an enantiomerically enriched sample of 97:3 er; colorless oil. The enantiomeric purity was established by HPLC analysis using a chiral column (Chiralpak IB column, $22{ }^{\circ} \mathrm{C}, 0.75 \mathrm{~mL} / \mathrm{min}, 96: 4$ Hexane:Isopropanol, $254 \mathrm{~nm}, \mathrm{t}_{\text {minor }}=13.013 \mathrm{~min}, \mathrm{t}_{\text {major }}=14.524 \mathrm{~min}$ ). Absolute stereochemistry was determined through analogy with S10. See supporting information part B for HPLC chromatograms.

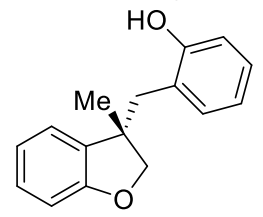


(+)-(S)-3-Methyl-3-(2-methylbenzyl)-2,3-dihydrobenzofuran (6): 6 was prepared according to general procedure above using 2-iodotoluene and was purified by silica gel column chromatography (Hex : DCM $=15: 1$ ) to obtain 6 as colorless oil (68\% yield, 97.5:2.5 er). 5\% direct cross-coupling byproduct was observed in the crude reaction mixture. IR (neat): 3019 (m), 2961 (s), 2926 (s), 2874 (m), 1596 (m), 1479 (s), 1458 (m), 975 (m), 746 (s); ${ }^{1} \mathbf{H}$ NMR (400 MHz, CDCl $)$ : $\delta$ 7.21-7.05 (m, 4H), 7.01 (d, J = 7.2 Hz, $1 \mathrm{H}), 6.87-6.76(\mathrm{~m}, 2 \mathrm{H}), 6.73(\mathrm{~d}, J=7.4 \mathrm{~Hz}, 1 \mathrm{H}), 4.46(\mathrm{~d}, J=8.5 \mathrm{~Hz}, 1 \mathrm{H}), 4.13(\mathrm{~d}, J=$ $8.5 \mathrm{~Hz}, 1 \mathrm{H}), 3.04(\mathrm{~d}, J=13.5 \mathrm{~Hz}, 1 \mathrm{H}), 2.84(\mathrm{~d}, J=13.6 \mathrm{~Hz}, 1 \mathrm{H}), 1.97$ (s, 3H), 1.41 (s, $3 \mathrm{H}) ;{ }^{13} \mathbf{C}$ NMR (100 MHz, $\left.\mathbf{C D C l}_{3}\right): \delta 159.7,137.6,136.1,134.8,131.1,130.5,128.3$, 126.7, 125.5, 123.6, 120.5, 109.8, 83.0, 46.9, 42.4, 24.3, 19.9; HRMS (CI+): Calcd for $\mathrm{C}_{17} \mathrm{H}_{19} \mathrm{O}\left(\mathrm{M}^{+}+\mathrm{H}\right): 239.1430$, Found 239.1425; Optical Rotation: $[\alpha]_{\mathrm{D}}{ }^{20}+43.3(c 0.75$ in $\mathrm{CHCl}_{3}$ ) for an enantiomerically enriched sample of 97:3 er. The enantiomeric purity was established by HPLC analysis using a chiral column (Chiralpak IA column, $22{ }^{\circ} \mathrm{C}, 0.3$ $\mathrm{mL} / \mathrm{min}$, 99:1 Hexane:Isopropanol, $254 \mathrm{~nm}, \mathrm{t}_{\text {minor }}=15.701 \mathrm{~min}, \mathrm{t}_{\text {major }}=17.035 \mathrm{~min}$ ). Absolute stereochemistry was determined through analogy with S10. See supporting information part B for HPLC chromatograms.

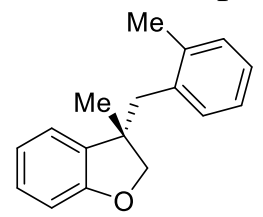

(+)-(S)-3-(2,6-Dimethylbenzyl)-3-methyl-2,3-dihydrobenzofuran (7): 7 was prepared according to general procedure above using 2-iodo-1,3-dimethylbenzene $(0.15 \mathrm{mmol}, 1.5$ equiv) and was purified by silica gel column chromatography (Hex : DCM = 10:1) to obtain 7 as colorless oil (68\% yield, 96:4 er). 4\% direct cross-coupling byproduct was observed in the crude reaction mixture. IR (neat): $3056(\mathrm{w}), 2962(\mathrm{~m}), 2876(\mathrm{~m}), 1596$ (m), 1479 (s), 1459 (m), 1378 (w), 973 (m), 751 (s); ${ }^{1} \mathbf{H}$ NMR (400 MHz, CDCl $)$ : $\delta$ 7.14 (app. td, $J=7.8,1.3 \mathrm{~Hz}, 1 \mathrm{H}), 7.03$ (dd, $J=8.4,6.3 \mathrm{~Hz}, 1 \mathrm{H}), 6.99-6.96$ (m, 2H), 6.81-6.76 (m, 2H), $6.70(\mathrm{dd}, J=7.6,1.2 \mathrm{~Hz}, 1 \mathrm{H}), 4.43(\mathrm{~d}, J=8.6 \mathrm{~Hz}, 1 \mathrm{H}), 4.14(\mathrm{~d}, J=$ $8.6 \mathrm{~Hz}, 1 \mathrm{H}), 3.11(\mathrm{~d}, J=14.0 \mathrm{~Hz}, 1 \mathrm{H}), 3.02(\mathrm{~d}, J=14.0 \mathrm{~Hz}, 1 \mathrm{H}), 2.02(\mathrm{~s}, 6 \mathrm{H}), 1.41$ (s, $3 \mathrm{H}) ;{ }^{13} \mathbf{C}$ NMR (100 MHz, $\left.\mathbf{C D C l}_{3}\right): \delta$ 159.6, 138.2, 135.3, 135.1, 128.4, 128.3, 126.3, 123.9, 120.6, 109.8, 83.6, 47.6, 38.9, 25.7, 21.0; HRMS (CI+): Calcd for $\mathrm{C}_{18} \mathrm{H}_{21} \mathrm{O}$ $\left(\mathrm{M}^{+}+\mathrm{H}\right): 253.1587$, Found 253.1592; Optical Rotation: $[\alpha]_{\mathrm{D}}{ }^{20}+43.3\left(c 0.75\right.$ in $\left.\mathrm{CHCl}_{3}\right)$ for an enantiomerically enriched sample of 96:4 er. The enantiomeric purity was established by HPLC analysis using a chiral column (Lux $3 \mathrm{u}$ Cellulose- 2 column, $22{ }^{\circ} \mathrm{C}$, $0.3 \mathrm{~mL} / \mathrm{min}, 99: 1$ Hexane:Isopropanol, $254 \mathrm{~nm}, \mathrm{t}_{\text {minor }}=13.427 \mathrm{~min}, \mathrm{t}_{\mathrm{major}}=14.504 \mathrm{~min}$ ). Absolute stereochemistry was determined through analogy with S10. See supporting information part B for HPLC chromatograms. 


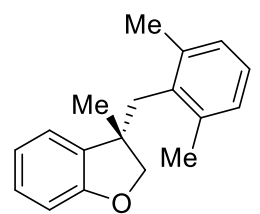

(+)-(S)-4-((3-Methyl-2,3-dihydrobenzofuran-3-yl)methyl)pyridine (8): 8 was prepared according to general procedure above using 4-iodopyridine and was purified by silica gel column chromatography (Hex : DCM : $\left.\mathrm{NEt}_{3}=5: 1: 0.1\right)$ to obtain 8 as colorless oil $(42 \%$ yield, 97:3 er). 9\% direct cross-coupling byproduct was observed in the crude reaction mixture. IR (neat): 3024 (w), 2987 (m), 2924 (s), 1598 (s), 1480 (s), 1354 (m), 1234 (m), 977 (w), 751 (s); ${ }^{\mathbf{H}} \mathbf{N M R}$ (400 $\mathbf{M H z}, \mathbf{C D C l}_{3}$ ): $\delta 8.45$ (app. br s, 2H), 7.15 (app. t, $J=$ $7.6 \mathrm{~Hz}, 1 \mathrm{H}), 6.94-6.84(\mathrm{~m}, 4 \mathrm{H}), 6.76(\mathrm{~d}, J=7.9 \mathrm{~Hz}, 1 \mathrm{H}), 4.45(\mathrm{~d}, J=8.8 \mathrm{~Hz}, 1 \mathrm{H}), 4.10$ (d, $J=8.8 \mathrm{~Hz}, 1 \mathrm{H}), 2.88(\mathrm{~d}, J=13.3 \mathrm{~Hz}, 1 \mathrm{H}), 2.84(\mathrm{~d}, J=13.5 \mathrm{~Hz}, 1 \mathrm{H}), 1.39(\mathrm{~s}, 3 \mathrm{H}) ;{ }^{13} \mathrm{C}$ NMR (100 MHz, $\left.\mathbf{C D C l}_{3}\right): \delta$ 159.6, 149.5, 146.5, 133.8, 128.8, 125.7, 123.4, 120.6, 110.1, 81.9, 46.3, 46.2, 24.6; HRMS (EI+): Calcd for $\mathrm{C}_{15} \mathrm{H}_{15} \mathrm{NO}\left(\mathrm{M}^{+}\right)$: 225.1148, Found 225.1158; Optical Rotation: $[\alpha]_{\mathrm{D}}{ }^{20}+4.8$ ( $c 0.375$ in $\mathrm{CHCl}_{3}$ ) for an enantiomerically enriched sample of 97:3 er. The enantiomeric purity was established by HPLC analysis using a chiral column (Lux $3 \mathrm{u}$ Cellulose-1 column, $22{ }^{\circ} \mathrm{C}, 0.75 \mathrm{~mL} / \mathrm{min}, 96: 4$ Hexane:Isopropanol, $254 \mathrm{~nm}, \mathrm{t}_{\text {minor }}=22.890 \mathrm{~min}, \mathrm{t}_{\text {major }}=26.471 \mathrm{~min}$ ). Absolute stereochemistry was determined through analogy with S10. See supporting information part B for HPLC chromatograms.

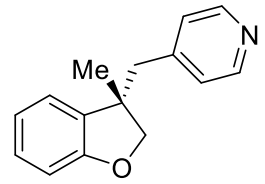

(-)-(S)-5-Methyl-2-((3-methyl-2,3-dihydrobenzofuran-3-yl)methyl)pyridine (9): 9 was prepared according to general procedure above using 2-bromo-5-methylpyridine and 5 mol\% $\mathrm{CuBr}-(R, R)-\mathrm{BenzP}$ and was purified by silica gel column chromatography (Hex : EtOAc $=5: 1)$ to obtain 9 as colorless oil $(63 \%$ yield, 97.5:2.5 er). 5\% direct crosscoupling byproduct was observed in the crude reaction mixture. IR (neat): 3004 (w), 2959 (m), 2924 (m), 1598 (m), 1481 (s), 1458 (m), 973 (m), 749 (s); ${ }^{1}$ H NMR (400 MHz, $\left.\mathbf{C D C l}_{3}\right): \delta 8.36(\mathrm{~s}, 1 \mathrm{H}), 7.31-7.24(\mathrm{~m}, 1 \mathrm{H}), 7.12$ (app. td, $\left.J=7.7,1.5 \mathrm{~Hz}, 1 \mathrm{H}\right), 7.04(\mathrm{~d}, J$ $=7.5 \mathrm{~Hz}, 1 \mathrm{H}), 6.87$ (app. t $J=7.4 \mathrm{~Hz}, 1 \mathrm{H}), 6.73(\mathrm{~d}, J=8.0 \mathrm{~Hz}, 1 \mathrm{H}), 6.68(\mathrm{~d}, J=7.9 \mathrm{~Hz}$, $1 \mathrm{H}), 4.76(\mathrm{~d}, J=8.8 \mathrm{~Hz}, 1 \mathrm{H}), 4.11(\mathrm{~d}, J=8.7 \mathrm{~Hz}, 1 \mathrm{H}), 3.06(\mathrm{~d}, J=13.2 \mathrm{~Hz}, 1 \mathrm{H}), 3.02(\mathrm{~d}$, $J=13.3 \mathrm{~Hz}, 1 \mathrm{H}), 2.28(\mathrm{~s}, 3 \mathrm{H}), 1.38(\mathrm{~s}, 3 \mathrm{H}) ;{ }^{\mathbf{1 3}} \mathbf{C}$ NMR (100 MHz, $\left.\mathbf{C D C l}_{3}\right): \delta$ 159.7, 155.4, 149.6, 136.5, 135.0, 130.9, 128.3, 124.0, 123.1, 120.5, 109.8, 81.8, 48.1, 46.3, 25.3, 18.2; HRMS (CI+): Calcd for $\mathrm{C}_{16} \mathrm{H}_{18} \mathrm{NO}\left(\mathrm{M}^{+}+\mathrm{H}\right)$ : 240.1383, Found 240.1385; Optical Rotation: $[\alpha]_{\mathrm{D}}{ }^{20}-32.1\left(c 0.375\right.$ in $\mathrm{CHCl}_{3}$ ) for an enantiomerically enriched sample of 97.5:2.5 er. The enantiomeric purity was established by HPLC analysis using a chiral column (Lux 3u Cellulose-1 column, $22{ }^{\circ} \mathrm{C}, 0.75 \mathrm{~mL} / \mathrm{min}, 96: 4$ Hexane:Isopropanol, 254 
$\left.\mathrm{nm}, \mathrm{t}_{\text {minor }}=8.893 \mathrm{~min}, \mathrm{t}_{\text {major }}=9.510 \mathrm{~min}\right)$. Absolute stereochemistry was determined through analogy with S10. See supporting information part B for HPLC chromatograms.

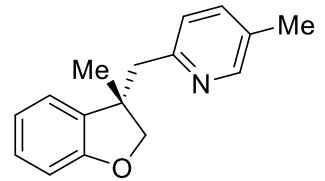

(-)-(S)-2-((3-Methyl-2,3-dihydrobenzofuran-3-yl)methyl)pyridine $\quad(\mathbf{1 0}): \quad \mathbf{1 0}$ was prepared according to general procedure above using 2-bromopyridine and 5 mol\% $\mathrm{CuBr}-(R, R)$-BenzP and was purified by silica gel column chromatography (Hex : EtOAc $=5: 1)$ to obtain 10 as colorless oil (55\% yield, 97.5:2.5 er). 5\% direct cross-coupling byproduct was observed in the crude reaction mixture. IR (neat): $3011(\mathrm{w}), 2957(\mathrm{~m})$, 2923 (s), 1591 (s), 1480 (s), 1236 (w), 973 (w), 751 (s); ${ }^{\mathbf{1}} \mathbf{H}$ NMR (400 MHz, CDCl $)$ : $\delta$ 8.54 (d, $J=4.8 \mathrm{~Hz}, 1 \mathrm{H}$ ), 7.48 (app. td, $J=7.6,2.7 \mathrm{~Hz}, 1 \mathrm{H}), 7.15-7.09$ (m, 2H), 7.04 (dd, $J=7.5,1.6 \mathrm{~Hz}, 1 \mathrm{H}), 6.92-6.83(\mathrm{~m}, 1 \mathrm{H}), 6.79(\mathrm{~d}, J=7.8 \mathrm{~Hz}, 1 \mathrm{H}), 6.73(\mathrm{~d}, J=8.0 \mathrm{~Hz}, 1 \mathrm{H})$, $4.78(\mathrm{~d}, J=8.8 \mathrm{~Hz}, 1 \mathrm{H}), 4.13(\mathrm{~d}, J=8.8 \mathrm{~Hz}, 1 \mathrm{H}), 3.10(\mathrm{~d}, J=13.3 \mathrm{~Hz}, 1 \mathrm{H}), 3.06(\mathrm{~d}, J=$ $13.3 \mathrm{~Hz}, 1 \mathrm{H}), 1.40$ (s, 3H); ${ }^{13} \mathbf{C}$ NMR (100 MHz, $\left.\mathbf{C D C l}_{3}\right): \delta 159.7,158.4,149.2,135.9$, $134.9,128.4,124.6,123.1,121.6,120.5,109.9,81.9,48.6,46.4,25.3$; HRMS (CI+): Calcd for $\mathrm{C}_{15} \mathrm{H}_{16} \mathrm{NO}\left(\mathrm{M}^{+}+\mathrm{H}\right)$ : 226.1226, Found 226.1226; Optical Rotation: $[\alpha]_{\mathrm{D}}{ }^{20}{ }_{-}$ 21.5 ( $c 0.375$ in $\mathrm{CHCl}_{3}$ ) for an enantiomerically enriched sample of 97.5:2.5 er. The enantiomeric purity was established by HPLC analysis using a chiral column (Lux $3 \mathrm{u}$ Cellulose-1 column, $22{ }^{\circ} \mathrm{C}, 0.75 \mathrm{~mL} / \mathrm{min}, 96: 4$ Hexane:Isopropanol, $254 \mathrm{~nm}, \mathrm{t}_{\text {major }}=$ $10.821 \mathrm{~min}, \mathrm{t}_{\text {minor }}=12.040 \mathrm{~min}$ ). Absolute stereochemistry was determined through analogy with S10. See supporting information part B for HPLC chromatograms.

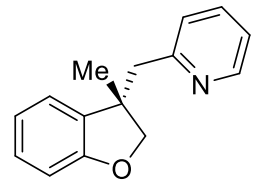

(+)-(S)-3-Methyl-2-((3-methyl-2,3-dihydrobenzofuran-3-yl)methyl)pyridine (11): 11 was prepared according to general procedure above using 1.5 equiv 2-bromo-3methylpyridine and $5 \mathrm{~mol} \% \mathrm{CuBr}-(R, R)$-BenzP and was purified by silica gel column chromatography (Hex : EtOAc $=5: 1$ ) to obtain 11 as colorless oil (69\% yield, 97:3 er). $7 \%$ direct cross-coupling byproduct was observed in the crude reaction mixture. IR (neat): 3052 (w), 2962 (m), 2924 (m), 2873 (w), 1597 (m), 1480 (s), 1450 (m), 970 (m), $747(\mathrm{~m}) ;{ }^{1} \mathbf{H}$ NMR (400 MHz, $\left.\mathbf{C D C l}_{3}\right): \delta 8.41(\mathrm{~d}, J=4.8 \mathrm{~Hz}, 1 \mathrm{H}), 7.34(\mathrm{~d}, J=7.4 \mathrm{~Hz}$, $1 \mathrm{H}), 7.19-7.08(\mathrm{~m}, 1 \mathrm{H}), 7.08-7.01(\mathrm{~m}, 1 \mathrm{H}), 6.84-6.70(\mathrm{~m}, 3 \mathrm{H}), 4.78(\mathrm{~d}, J=8.9 \mathrm{~Hz}, 1 \mathrm{H})$, $4.26(\mathrm{~d}, J=9.0 \mathrm{~Hz}, 1 \mathrm{H}), 3.22$ (d, $J=14.2 \mathrm{~Hz}, 1 \mathrm{H}), 3.00(\mathrm{~d}, J=13.9 \mathrm{~Hz}, 1 \mathrm{H}), 1.94(\mathrm{~s}, 3 \mathrm{H})$, 1.45 (s, 3H); ${ }^{13} \mathbf{C}$ NMR (100 MHz, $\left.\mathbf{C D C l}_{3}\right): \delta 159.7,157.1,146.5,137.7,135.4,132.7$, 128.3, 123.2, 121.6, 120.4, 109.8, 83.4, 46.4, 44.2, 24.4, 19.1; HRMS (CI+): Calcd for $\mathrm{C}_{16} \mathrm{H}_{18} \mathrm{NO}\left(\mathrm{M}^{+}+\mathrm{H}\right): 240.1383$, Found 240.1378; Optical Rotation: $[\alpha]_{\mathrm{D}}{ }^{20}+36.6(c 0.375$ 
in $\mathrm{CHCl}_{3}$ ) for an enantiomerically enriched sample of 97:3 er. The enantiomeric purity was established by HPLC analysis using a chiral column (Lux 3u Cellulose-1 column, $22{ }^{\circ} \mathrm{C}, 0.75 \mathrm{~mL} / \mathrm{min}, 96: 4$ Hexane:Isopropanol, $254 \mathrm{~nm}, \mathrm{t}_{\text {major }}=7.580 \mathrm{~min}, \mathrm{t}_{\text {minor }}=8.090$ min). Absolute stereochemistry was determined through analogy with S10. See supporting information part B for HPLC chromatograms.

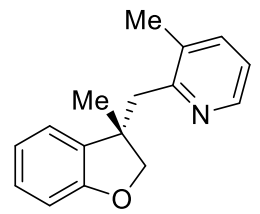

(+)-(S)-1-((3-Methyl-2,3-dihydrobenzofuran-3-yl)methyl)isoquinoline (12): 12 was prepared according to general procedure above using 1.5 equiv 1-bromoisoquinoline and $5 \mathrm{~mol} \% \mathrm{CuBr}-(R, R)$-BenzP and was purified by silica gel column chromatography (Hex : EtOAc $=5: 1)$ to obtain 12 as colorless oil (58\% yield, 98:2 er). 7\% direct cross-coupling byproduct was observed in the crude reaction mixture. IR (neat): $3055(\mathrm{~m}), 2963(\mathrm{~m})$, 2924 (m), 1597 (m), 1480 (s), 1458 (m), 1219 (m), 970 (m), 750 (s); ${ }^{1}$ H NMR (400 MHz, $\left.\mathbf{C D C l}_{3}\right): \delta 8.49(\mathrm{~d}, J=5.6 \mathrm{~Hz}, 1 \mathrm{H}), 7.88(\mathrm{~d}, J=8.5 \mathrm{~Hz}, 1 \mathrm{H}), 7.78(\mathrm{~d}, J=8.1 \mathrm{~Hz}, 1 \mathrm{H})$, 7.61 (app. t, $J=7.4 \mathrm{~Hz}, 1 \mathrm{H}), 7.54$ (d, $J=5.5 \mathrm{~Hz}, 1 \mathrm{H}$ ), 7.44 (app. t, $J=7.8 \mathrm{~Hz}, 1 \mathrm{H}$ ), 7.10 (app. t, $J=7.5 \mathrm{~Hz}, 1 \mathrm{H}), 6.96(\mathrm{~d}, J=7.1 \mathrm{~Hz}, 1 \mathrm{H}), 6.84-6.73(\mathrm{~m}, 2 \mathrm{H}), 4.87(\mathrm{~d}, J=9.1 \mathrm{~Hz}$, $1 \mathrm{H}), 4.27$ (d, $J=9.1 \mathrm{~Hz}, 1 \mathrm{H}), 3.75$ (d, $J=14.6 \mathrm{~Hz}, 1 \mathrm{H}), 3.51(\mathrm{~d}, J=14.3 \mathrm{~Hz}, 1 \mathrm{H}), 1.48$ (s, $3 \mathrm{H}) ;{ }^{13} \mathbf{C}$ NMR (100 MHz, $\left.\mathbf{C D C l}_{3}\right): \delta 159.5,158.5,141.8,136.2,135.7,129.8,128.4$, $128.3,127.4,127.0,125.5,123.2,120.5,119.7,109.9,82.9,46.5,43.8,24.7$; HRMS (CI+): Calcd for $\mathrm{C}_{15} \mathrm{H}_{15} \mathrm{NO}\left(\mathrm{M}^{+}+\mathrm{H}\right)$ : 276.1383, Found 276.1380; Optical Rotation: $[\alpha]_{\mathrm{D}}^{20}+38.2\left(c 0.375\right.$ in $\left.\mathrm{CHCl}_{3}\right)$ for an enantiomerically enriched sample of 98:2 er. The enantiomeric purity was established by HPLC analysis using a chiral column (Lux $3 \mathrm{u}$ Cellulose-1 column, $22{ }^{\circ} \mathrm{C}, 0.75 \mathrm{~mL} / \mathrm{min}, 96: 4$ Hexane:Isopropanol, $254 \mathrm{~nm}, \mathrm{t}_{\text {major }}=$ $\left.8.911 \mathrm{~min}, \mathrm{t}_{\text {major }}=9.576 \mathrm{~min}\right)$. Absolute stereochemistry was determined through analogy with S10. See supporting information part B for HPLC chromatograms.

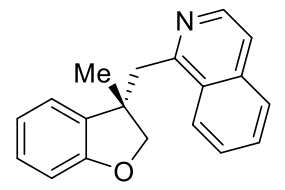

(+)-(S)-3-Benzyl-6-methoxy-3-methyl-2,3-dihydrobenzofuran (13): 13 was prepared according to general procedure above using ArBpin (S1) and iodobenzene and was purified by silica gel column chromatography $(\mathrm{Hex}: \mathrm{DCM}=2: 1$ ) to obtain $\mathbf{1 3}$ as colorless oil (71\% yield, 98:2 er). 9\% direct cross-coupling byproduct was observed in the crude reaction mixture. IR (neat): 3061 (w), 3027 (m), 2959 (s), 2925 (s), 2835 (m), 1621 (s), 1597 (s), 1281 (s), 1146 (s), 1027 (m), 703 (s); ${ }^{\mathbf{1}} \mathbf{H}$ NMR (400 MHz, CDCl $)$ : $\delta$ 7.28-7.21 (m, 3H), 7.03-6.97 (m, 2H), $6.79(\mathrm{~d}, J=8.2 \mathrm{~Hz}, 1 \mathrm{H}), 6.42(\mathrm{dd}, J=8.2,2.2 \mathrm{~Hz}$, $1 \mathrm{H}), 6.36(\mathrm{~d}, J=2.1 \mathrm{~Hz}, 1 \mathrm{H}), 4.51(\mathrm{~d}, J=8.7 \mathrm{~Hz}, 1 \mathrm{H}), 4.09(\mathrm{~d}, J=8.7 \mathrm{~Hz}, 1 \mathrm{H}), 3.77$ (s, 
3H), $2.88(\mathrm{~d}, J=13.2 \mathrm{~Hz}, 1 \mathrm{H}), 2.84(\mathrm{~d}, J=13.2 \mathrm{~Hz}, 1 \mathrm{H}), 1.33(\mathrm{~s}, 3 \mathrm{H}) ;{ }^{13} \mathbf{C}$ NMR $(\mathbf{1 0 0}$ MHz, $\left.\mathbf{C D C l}_{3}\right): \delta 160.9,160.6,137.8,130.5,128.0,127.1,126.5,123.6,106.0,96.4,82.9$, 55.6, 47.0, 45.9, 24.9; HRMS (EI+): Calcd for $\mathrm{C}_{17} \mathrm{H}_{18} \mathrm{O}_{2}\left(\mathrm{M}^{+}\right)$: 254.1301, Found 254.1313; Optical Rotation: $[\alpha]_{\mathrm{D}}{ }^{20}+24.3\left(c 0.75\right.$ in $\left.\mathrm{CHCl}_{3}\right)$ for an enantiomerically enriched sample of 98:2 er. The enantiomeric purity was established by HPLC analysis using a chiral column (Chiralpak IA column, $22 \quad{ }^{\circ} \mathrm{C}, \quad 0.3 \mathrm{~mL} / \mathrm{min}, \quad 99: 1$ Hexane:Isopropanol, $254 \mathrm{~nm}, \mathrm{t}_{\text {major }}=24.901 \mathrm{~min}, \mathrm{t}_{\text {minor }}=26.566 \mathrm{~min}$ ). Absolute stereochemistry was determined through analogy with S10. See supporting information part B for HPLC chromatograms.

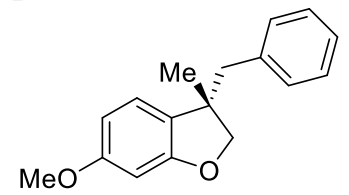

(+)-(S)-t-Butyl(4-((6-chloro-3-methyl-2,3-dihydrobenzofuran-3-yl)methyl)phenoxy)

dimethylsilane (14): 14 was prepared according to general procedure above using ArBpin (21) and $t$-butyl(4-iodophenoxy)dimethylsilane and was purified by silica gel column chromatography (Hex : DCM = 5:1) to obtain 14 as colorless oil (49\% yield, 97:3 er). $10 \%$ direct cross-coupling byproduct was observed in the crude reaction mixture. IR (neat): 3030 (w), 2957 (s), 2929 (s), 2857 (m), 1607 (m), 1509 (s), 1260 (s), 977 (m), 916 (s), $781(\mathrm{~m}) ;{ }^{1} \mathbf{H}$ NMR (400 MHz, $\left.\mathbf{C D C l}_{3}\right): \delta$ 6.85-6.78 (m, 3H), 6.76-6.69 (m, 4H), 4.50 $(\mathrm{d}, J=8.7 \mathrm{~Hz}, 1 \mathrm{H}), 4.10(\mathrm{~d}, J=8.7 \mathrm{~Hz}, 1 \mathrm{H}), 2.80(\mathrm{~d}, J=13.5 \mathrm{~Hz}, 1 \mathrm{H}), 2.76(\mathrm{~d}, J=13.5$ Hz, 1H), 1.32 (s, 3H), 0.98 (s, 9H), 0.18 (s, 6H); ${ }^{\mathbf{1 3}} \mathbf{C}$ NMR (100 MHz, CDCl $\left.\mathbf{3}\right): \delta 160.6$, 154.5, 133.6, 133.5, 131.4, 130.0, 124.2, 120.4, 119.7, 110.6, 83.0, 46.2, 46.0, 25.8, 24.6, 18.4, -4.3; HRMS (EI+): Calcd for $\mathrm{C}_{22} \mathrm{H}_{29} \mathrm{ClO}_{2} \mathrm{Si}\left(\mathrm{M}^{+}\right)$: 388.1620, Found 388.1611; Optical Rotation: $[\alpha]_{\mathrm{D}}^{20}+40.7\left(c 0.75\right.$ in $\mathrm{CHCl}_{3}$ ) for an enantiomerically enriched sample of 97:3 er. Enantiopurity was determined through deprotection to phenol S7 (see below). Absolute stereochemistry was determined through analogy with S10.

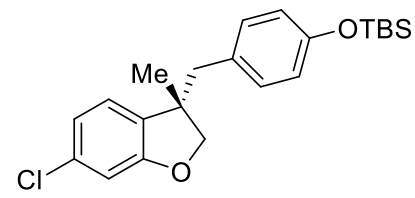

(S)-4-((6-Chloro-3-methyl-2,3-dihydrobenzofuran-3-yl)methyl)phenol (S7): S7 was prepared from $\mathbf{1 4}$ according to modified literature procedure. ${ }^{12}$ A solution of the silyl ether $14(0.1 \mathrm{mmol}, 1.0$ equiv) in dry THF $(1 \mathrm{~mL})$ was treated with tetrabutylammonium fluoride (1.0 M solution in THF, $0.3 \mathrm{mmol}, 3.0$ equiv), and was allowed to stir at room temperature for $3 \mathrm{~h}$. The reaction was quenched with $\mathrm{H}_{2} \mathrm{O}(2 \mathrm{~mL})$ and brine $(2 \mathrm{~mL})$ and the mixture was extracted with $\mathrm{Et}_{2} \mathrm{O}(3 \times 3 \mathrm{~mL})$. The combined organic layers were dried over $\mathrm{MgSO}_{4}$, filtered, and concentrated. The residue was purified by silica gel column chromatography $\left(\mathrm{Hex}_{\mathrm{E}} \mathrm{Et}_{2} \mathrm{O}=1: 1\right)$ to obtain the desired product in $91 \%$ yield. ${ }^{1} \mathbf{H}$ NMR 
(400 MHz, CDCl $\left.\mathbf{~}_{3}\right): \delta 6.89-6.66(\mathrm{~m}, 7 \mathrm{H}), 4.74($ br s, $1 \mathrm{H}), 4.49(\mathrm{~d}, J=8.7 \mathrm{~Hz}, 1 \mathrm{H}), 4.10$ $(\mathrm{d}, J=8.7 \mathrm{~Hz}, 1 \mathrm{H}), 2.81(\mathrm{~d}, J=13.5 \mathrm{~Hz}, 1 \mathrm{H}), 2.76(\mathrm{~d}, J=13.5 \mathrm{~Hz}, 1 \mathrm{H}), 1.33(\mathrm{~s}, 3 \mathrm{H}) ;{ }^{13} \mathbf{C}$ NMR (100 MHz, $\left.\mathbf{C D C l}_{3}\right): \delta 160.6,154.4,133.6,131.6,129.5,124.2,120.5,115.0$, $110.6,82.9,46.1,45.9,24.7$; colorless oil. The enantiomeric purity was established by HPLC analysis using a chiral column (Chiralpak IB column, $22{ }^{\circ} \mathrm{C}, 0.3 \mathrm{~mL} / \mathrm{min}, 96: 4$ Hexane:Isopropanol, $254 \mathrm{~nm}, \mathrm{t}_{\text {major }}=15.891 \mathrm{~min}, \mathrm{t}_{\text {minor }}=17.487 \mathrm{~min}$ ). Absolute stereochemistry was determined through analogy with S10. See supporting information part B for HPLC chromatograms.

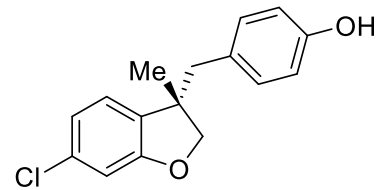

(-)-(S)-3-Benzyl-3-butyl-2,3-dihydrobenzofuran (15): 15 was prepared according to general procedure above using ArBpin (S3) and iodobenzene and was purified by silica gel column chromatography (Hex : DCM =10:1) to obtain 15 as colorless oil (70\% yield, 97:3 er). $5 \%$ direct cross-coupling byproduct was observed in the crude reaction mixture. IR (neat): 3083 (w), 3061 (m), 2930 (s), 2859 (s), 1597 (s), 1481 (s), 1231 (m), 1019 (m), 974 (s), 750 (s), 701 (s); ${ }^{1} \mathbf{H}$ NMR (400 MHz, $\mathbf{C D C l}_{3}$ ): $\delta$ 7.24-7.18 (m, 3H), 7.13 (app. t, $J=7.6 \mathrm{~Hz}, 1 \mathrm{H}), 6.98-6.94(\mathrm{~m}, 2 \mathrm{H}), 6.92$ (d, $J=7.4 \mathrm{~Hz}, 1 \mathrm{H}), 6.86$ (app. t, $J=7.3 \mathrm{~Hz}, 1 \mathrm{H}$ ), $6.73(\mathrm{~d}, J=8.0 \mathrm{~Hz}, 1 \mathrm{H}), 4.45(\mathrm{~d}, J=8.8 \mathrm{~Hz}, 1 \mathrm{H}), 4.23(\mathrm{~d}, J=8.8 \mathrm{~Hz}, 1 \mathrm{H}), 2.99(\mathrm{~d}, J=$ $13.4 \mathrm{~Hz}, 1 \mathrm{H}), 2.92$ (d, $J=13.4 \mathrm{~Hz}, 1 \mathrm{H}), 1.75-1.64(\mathrm{~m}, 2 \mathrm{H}), 1.42-1.20$ (m, 3H), 1.19-1.05 $(\mathrm{m}, 1 \mathrm{H}), 0.87(\mathrm{t}, J=7.1 \mathrm{~Hz}, 3 \mathrm{H}) ;{ }^{13} \mathbf{C} \mathbf{N M R}\left(\mathbf{1 0 0} \mathbf{M H z}, \mathbf{C D C l}_{3}\right): \delta 160.2,137.6,133.2$, $130.5,128.3,128.0,126.5,124.1,120.2$, 109.6, 80.1, 50.0, 45.2, 37.7, 26.8, 23.4, 14.2; HRMS (CI+): Calcd for $\mathrm{C}_{19} \mathrm{H}_{23} \mathrm{O}\left(\mathrm{M}^{+}+\mathrm{H}\right)$ : 267.1743, Found 267.1740; Optical Rotation: $[\alpha]_{\mathrm{D}}{ }^{20}-8.2\left(c 0.75\right.$ in $\left.\mathrm{CHCl}_{3}\right)$ for an enantiomerically enriched sample of 97:3 er. The enantiomeric purity was established by HPLC analysis using a chiral column (Lux 3u Cellulose- 1 column, $22^{\circ} \mathrm{C}, 0.5 \mathrm{~mL} / \mathrm{min}, 99: 1$ Hexane:Isopropanol, $254 \mathrm{~nm}, \mathrm{t}_{\text {minor }}$ $\left.=10.040 \mathrm{~min}, \mathrm{t}_{\text {major }}=11.052 \mathrm{~min}\right)$. Absolute stereochemistry was determined through analogy with S10. See supporting information part B for HPLC chromatograms.

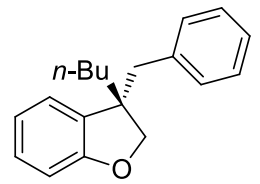

(-)-(R)-3-Benzyl-3-isopropyl-2,3-dihydrobenzofuran (16): 16 was prepared according to general procedure above using ArBpin (S4) and iodobenzene and was purified by silica gel column chromatography (Hex : $\mathrm{DCM}=10: 1)$ to obtain 16 as colorless oil $(52 \%$ yield, 96:4 er). $6 \%$ direct cross-coupling byproduct was observed in the crude reaction mixture. IR (neat): 3061 (w), 3029 (m), 2960 (s), 2929 (s), 1595 (m), 1482 (s), 1458 (s), 1235 (m), 1020 (m), 971 (m), 750 (s), 701 (s); ${ }^{1} \mathbf{H}$ NMR (400 MHz, $\mathbf{C D C l}_{3}$ ): $\delta$ 7.19-7.13 
(app. br s, 3H), 7.10 (app. t, $J=7.6 \mathrm{~Hz}, 1 \mathrm{H}), 6.93(\mathrm{~d}, J=7.3 \mathrm{~Hz}, 1 \mathrm{H}), 6.90-6.80$ (m, 3H), 6.67 (d, $J=7.9 \mathrm{~Hz}, 1 \mathrm{H}), 4.35$ (app. s, 2H), 3.06 (d, $J=13.6 \mathrm{~Hz}, 1 \mathrm{H}), 3.00$ (d, $J=13.5 \mathrm{~Hz}$, 1H), 2.08 (hept, $J=6.7 \mathrm{~Hz}, 1 \mathrm{H}), 0.99$ (d, $J=6.7 \mathrm{~Hz}, 3 \mathrm{H}), 0.89(\mathrm{~d}, J=6.7 \mathrm{~Hz}, 3 \mathrm{H}) ;{ }^{13} \mathrm{C}$ NMR (100 MHz, $\left.\mathbf{C D C l}_{3}\right): \delta$ 160.6, 137.7, 131.4, 130.5, 128.3, 128.0, 126.5, 125.0, 119.8, 109.5, 77.6, 53.8, 43.4, 33.5, 18.3, 17.9; HRMS (CI+): Calcd for $\mathrm{C}_{18} \mathrm{H}_{21} \mathrm{O}$ $\left(\mathrm{M}^{+}+\mathrm{H}\right): 253.1587$, Found 253.1588; Optical Rotation: $[\alpha]_{\mathrm{D}}{ }^{20}-50.0\left(c 0.75\right.$ in $\left.\mathrm{CHCl}_{3}\right)$ for an enantiomerically enriched sample of 96:4 er. The enantiomeric purity was established by HPLC analysis using a chiral column (Chiralpak IB column, $22{ }^{\circ} \mathrm{C}, 0.3$ $\mathrm{mL} / \mathrm{min}, 99: 1$ Hexane:Isopropanol, $254 \mathrm{~nm}, \mathrm{t}_{\text {minor }}=16.298 \mathrm{~min}, \mathrm{t}_{\mathrm{major}}=17.304 \mathrm{~min}$ ). Absolute stereochemistry was determined through analogy with S10. See supporting information part B for HPLC chromatograms.

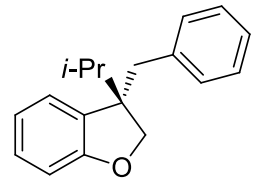

(-)-(S)-3-Benzyl-3-(3-methylbut-3-en-1-yl)-2,3-dihydrobenzofuran $\quad(17): \quad 17$ was prepared according to general procedure above using ArBpin (S2) and iodobenzene and was purified by silica gel column chromatography (Hex : $\mathrm{DCM}=10: 1$ ) to obtain 17 as colorless oil (69\% yield, 97:3 er). 7\% direct cross-coupling byproduct was observed in the crude reaction mixture. IR (neat): 3065 (w), 3028 (m), 2925 (s), 2854 (m), 1597 (m), 1480 (s), 1458 (s), 1232 (m), 978 (s), 761 (s), 702 (s); ${ }^{\mathbf{1}}$ H NMR (400 MHz, CDCl 3 ): $\delta$ 7.24-7.18 (m, 3H), 7.14 (app. t, $J=7.6 \mathrm{~Hz}, 1 \mathrm{H}), 6.99-6.91$ (m, 3H), 6.87 (app. t, $J=7.3$ $\mathrm{Hz}, 1 \mathrm{H}), 6.74(\mathrm{~d}, J=8.0 \mathrm{~Hz}, 1 \mathrm{H}), 4.69(\mathrm{~s}, 1 \mathrm{H}), 4.65(\mathrm{~s}, 1 \mathrm{H}), 4.47$ (d, J = 8.9 Hz, 1H), $4.24(\mathrm{~d}, J=8.9 \mathrm{~Hz}, 1 \mathrm{H}), 3.01(\mathrm{~d}, J=13.4 \mathrm{~Hz}, 1 \mathrm{H}), 2.95(\mathrm{~d}, J=13.4 \mathrm{~Hz}, 1 \mathrm{H}), 2.10-2.08$ $(\mathrm{m}, 1 \mathrm{H}), 1.90-1.76(\mathrm{~m}, 3 \mathrm{H}), 1.68(\mathrm{~s}, 3 \mathrm{H}) ;{ }^{13} \mathbf{C}$ NMR (100 MHz, $\left.\mathbf{C D C l}_{3}\right): \delta 160.2,145.7$, 137.4, 132.8, 130.5, 128.4, 128.1, 126.6, 124.1, 120.3, 110.0, 109.7, 79.8, 49.9, 45.3, 36.1, 32.7, 22.9; HRMS (EI+): Calcd for $\mathrm{C}_{20} \mathrm{H}_{22} \mathrm{O}\left(\mathrm{M}^{+}\right)$: 278,1665, Found 278.1653; Optical Rotation: $[\alpha]_{\mathrm{D}}{ }^{20}-13.7\left(c 0.75\right.$ in $\mathrm{CHCl}_{3}$ ) for an enantiomerically enriched sample of 97:3 er. The enantiomeric purity was established by HPLC analysis using a chiral column (Chiralpak IB column, $22{ }^{\circ} \mathrm{C}, 0.3 \mathrm{~mL} / \mathrm{min}, 99: 1$ Hexane:Isopropanol, $254 \mathrm{~nm}, \mathrm{t}_{\text {minor }}=$ $\left.15.278 \mathrm{~min}, \mathrm{t}_{\text {major }}=16.007 \mathrm{~min}\right)$. Absolute stereochemistry was determined through analogy with S10. See supporting information part B for HPLC chromatograms.

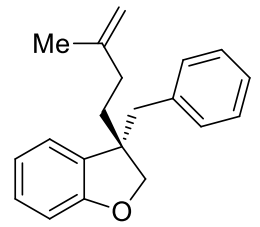

(+)-(S)-3-Benzyl-2,3-dihydrobenzofuran (18): 18 was prepared according to general procedure above using 2-(2-(allyloxy)phenyl)-4,4,5,5-tetramethyl-1,3,2-dioxaborolane ${ }^{4}$, 10 equiv iodobenzene and $1 \% \mathrm{CuBr}-(R, R)$-BenzP at $140{ }^{\circ} \mathrm{C}$ and was purified by silica gel 
column chromatography (Hex : DCM $=10: 1)$ to obtain 18 as colorless oil (52\% yield, 97:3 er). $10 \%$ direct cross-coupling byproduct was observed in the crude reaction mixture. IR (neat): 3062 (w), 3027 (m), 2924 (m), 1597 (m), 1481 (s), 1460 (m), 1231 (s), 967 (m), 747 (s); ${ }^{\mathbf{1}} \mathbf{H}$ NMR (400 MHz, $\left.\mathbf{C D C l}_{3}\right): \delta 7.32$ (t, $\left.J=7.2 \mathrm{~Hz}, 2 \mathrm{H}\right)$, 7.28-7.17 (m, 3H), 7.14 (app. t, $J=7.7 \mathrm{~Hz}, 1 \mathrm{H}), 6.98(\mathrm{~d}, J=7.2 \mathrm{~Hz}, 1 \mathrm{H}), 6.86-6.78(\mathrm{~m}, 2 \mathrm{H}), 4.53$ (app. t, $J=8.9 \mathrm{~Hz}, 1 \mathrm{H}), 4.32-4.26(\mathrm{~m}, 1 \mathrm{H}), 3.82-3.71(\mathrm{~m}, 1 \mathrm{H}), 3.07$ (dd, $J=13.8,6.1 \mathrm{~Hz}$, 1H), $2.85(\mathrm{dd}, J=13.5,9.2 \mathrm{~Hz}, 1 \mathrm{H}) ;{ }^{13} \mathbf{C} \mathbf{N M R}\left(\mathbf{1 0 0} \mathbf{M H z}, \mathbf{C D C l}_{3}\right): \delta 160.1,139.3$, 130.4, 129.1, 128.7, 128.5, 126.6, 124.7, 120.4, 109.8, 76.5, 43.6, 41.2; HRMS (EI+): Calcd for $\mathrm{C}_{15} \mathrm{H}_{14} \mathrm{O}\left(\mathrm{M}^{+}\right)$: 210.1039, Found 210.1035; Optical Rotation: $[\alpha]_{\mathrm{D}}{ }^{20}+35.7(c$ 0.75 in $\mathrm{CHCl}_{3}$ ) for an enantiomerically enriched sample of 97:3 er oil. The enantiomeric purity was established by HPLC analysis using a chiral column (Chiralpak IB column, $22{ }^{\circ} \mathrm{C}, 0.5 \mathrm{~mL} / \mathrm{min}, 99: 1$ Hexane:Isopropanol, $254 \mathrm{~nm}, \mathrm{t}_{\text {major }}=10.914 \mathrm{~min}, \mathrm{t}_{\text {minor }}=11.468$ min). Absolute stereochemistry was determined through analogy with S10. See supporting information part B for HPLC chromatograms.

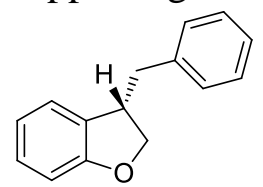

(+)-(S)-1,3-Dibenzyl-3-methylindoline (19): 19 was prepared according to general procedure above using ArBpin (S5), iodobenzene and 5\% $\mathrm{CuBr}-(R, R)$-BenzP catalyst and was purified by silica gel column chromatography (Hex : $\mathrm{DCM}=8: 1$ ) to obtain 19 as colorless oil (54\% yield, 93:7 er). 7\% direct cross-coupling byproduct was observed in the crude reaction mixture. IR (neat): 3060 (w), 3026 (s), 2958 (s), 2922 (s), 2822 (m), 1604 (s), 1492 (s), 1452 (s), 1358 (m), 739 (s), 699 (s); ${ }^{\mathbf{1}} \mathbf{H}$ NMR (400 MHz, CDCl $)$ ): $\delta$ 7.39-7.18 (m, 8H), 7.09 (app. t, $J=7.6 \mathrm{~Hz}, 1 \mathrm{H}$ ), 6.98 (app. br s, 2H), 6.93 (d, $J=7.2 \mathrm{~Hz}$, $1 \mathrm{H}), 6.70$ (app. t, $J=7.3 \mathrm{~Hz}, 1 \mathrm{H}), 6.47$ (d, $J=7.8 \mathrm{~Hz}, 1 \mathrm{H}), 4.34$ (d, $J=15.0 \mathrm{~Hz}, 1 \mathrm{H})$, $4.07(\mathrm{~d}, J=15.0 \mathrm{~Hz}, 1 \mathrm{H}), 3.34(\mathrm{~d}, J=8.8 \mathrm{~Hz}, 1 \mathrm{H}), 2.91-2.86(\mathrm{~m}, 2 \mathrm{H}), 2.83(\mathrm{~d}, J=13.4$ $\mathrm{Hz}, 1 \mathrm{H}), 1.32$ (s, 3H); ${ }^{13} \mathbf{C}$ NMR (100 $\left.\mathbf{~ M H z}, \mathbf{C D C l}_{3}\right): \delta 151.7,138.7,138.6,137.4,130.6$, 128.6, 127.9, 127.8, 127.2, 126.2, 122.9, 117.6, 107.2, 65.1, 53.2, 46.6, 44.8, 24.9; HRMS (EI+): Calcd for $\mathrm{C}_{23} \mathrm{H}_{23} \mathrm{~N}\left(\mathrm{M}^{+}\right)$: 313.1825, Found 313.1816; Optical Rotation: $[\alpha]_{\mathrm{D}}{ }^{20}+41.3\left(c 0.75\right.$ in $\left.\mathrm{CHCl}_{3}\right)$ for an enantiomerically enriched sample of 93:7 er. The enantiomeric purity was established by HPLC analysis using a chiral column (Lux $3 \mathrm{u}$ Cellulose-1 column, $22{ }^{\circ} \mathrm{C}, 0.3 \mathrm{~mL} / \mathrm{min}, 99: 1$ Hexane:Isopropanol, $254 \mathrm{~nm}, \mathrm{t}_{\text {minor }}=$ $27.841 \mathrm{~min}, \mathrm{t}_{\text {major }}=29.529 \mathrm{~min}$ ). Absolute stereochemistry was determined through analogy with S10. See supporting information part B for HPLC chromatograms.

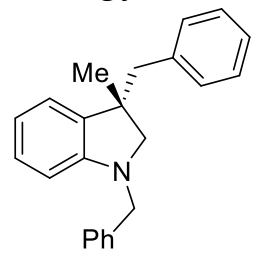


(+)-(S)-1,3-Dibenzylindoline (20): 20 was prepared according to general procedure above using $N$-allyl- $N$-benzyl-2-(4,4,5,5-tetramethyl-1,3,2-dioxaborolan-2-yl)aniline ${ }^{4}, 10$ equiv iodobenzene and $1 \% \mathrm{CuBr}-(R, R)$-BenzP at $140{ }^{\circ} \mathrm{C}$ and was purified by silica gel column chromatography (Hex : DCM $=10: 1)$ to obtain 20 as colorless oil (50\% yield, 89:11 er). $10 \%$ direct cross-coupling byproduct was observed in the crude reaction mixture. ${ }^{1} \mathbf{H}$ NMR (400 MHz, $\mathbf{C D C l}_{3}$ ): $\delta$ 7.37-7.13 (m, 10H), 7.08 (app. t, $J=7.8 \mathrm{~Hz}$, $1 \mathrm{H}), 6.97$ (d, $J=7.1 \mathrm{~Hz}, 1 \mathrm{H}), 6.66$ (app. t, $J=7.4 \mathrm{~Hz}, 1 \mathrm{H}), 6.50$ (d, $J=7.8 \mathrm{~Hz}, 1 \mathrm{H}), 4.27$ $(\mathrm{d}, J=15.1 \mathrm{~Hz}, 1 \mathrm{H}), 4.20$ (d, $J=15.2 \mathrm{~Hz}, 1 \mathrm{H}), 3.62-3.48$ (m, 1H), 3.34 (app. t, $J=8.8$ $\mathrm{Hz}, 1 \mathrm{H}), 3.16-2.99(\mathrm{~m}, 2 \mathrm{H}), 2.80(\mathrm{dd}, J=13.6,9.4 \mathrm{~Hz}, 1 \mathrm{H}) ;{ }^{13} \mathbf{C}$ NMR (100 MHz, $\left.\mathbf{C D C l}_{3}\right): \delta 152.4,140.2,138.6,133.1,129.2,128.6,128.5,127.9,127.9,127.2,126.3$, 124.1, 117.7, 107.2, 59.3, 53.4, 42.4, 40.6; Optical Rotation: $[\alpha]_{\mathrm{D}}{ }^{20}+45.4\left(c 1\right.$ in $\left.\mathrm{CHCl}_{3}\right)$ for an enantiomerically enriched sample of 89:11 er (lit. ${ }^{13}-34.6$ ( $c 1$ in $\mathrm{CH}_{2} \mathrm{Cl}_{2}$ ) for the opposite enantiomer, 80:20 er). The enantiomeric purity was established by HPLC analysis using a chiral column (Lux $3 \mathrm{u}$ Cellulose-1 column, $22{ }^{\circ} \mathrm{C}, 0.3 \mathrm{~mL} / \mathrm{min}, 99: 1$ Hexane:Isopropanol, $254 \mathrm{~nm}, \mathrm{t}_{\text {major }}=34.096 \mathrm{~min}, \mathrm{t}_{\text {minorr }}=37.098 \mathrm{~min}$ ). Absolute stereochemistry matched with the analogy from S10. See supporting information part B for HPLC chromatograms.

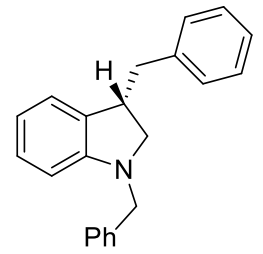

(+)-(S)-3-Benzyl-6-chloro-3-methyl-2,3-dihydrobenzofuran (22): 22 was prepared according to general procedure above using ArBpin (21) and iodobenzene and was purified by silica gel column chromatography (Hex : DCM = 10:1) to obtain 22 as colorless oil (51\% yield, 96:4 er). 9\% direct cross-coupling byproduct was observed in the crude reaction mixture. IR (neat): 3063 (w), 2959 (m), 2924 (m), 1602 (m), 1481 (s), 1417 (m), 977 (s), 876 (m), 702 (s); ${ }^{1} \mathbf{H}$ NMR (400 MHz, CDCl $): \delta$ 7.29-7.21 (m, 3H), $7.02-6.96(\mathrm{~m}, 2 \mathrm{H}), 6.84(\mathrm{dd}, J=7.9,1.8 \mathrm{~Hz}, 1 \mathrm{H}), 6.79(\mathrm{~d}, J=7.9 \mathrm{~Hz}, 1 \mathrm{H}), 6.76(\mathrm{~d}, J=$ $1.8 \mathrm{~Hz}, 1 \mathrm{H}), 4.53(\mathrm{~d}, J=8.8 \mathrm{~Hz}, 1 \mathrm{H}), 4.11(\mathrm{~d}, J=8.8 \mathrm{~Hz}, 1 \mathrm{H}), 2.89$ (d, $J=13.3 \mathrm{~Hz}, 1 \mathrm{H})$, $2.84(\mathrm{~d}, J=13.3 \mathrm{~Hz}, 1 \mathrm{H}), 1.35(\mathrm{~s}, 3 \mathrm{H}) ;{ }^{13} \mathbf{C}$ NMR (100 MHz, $\left.\mathbf{C D C l}_{3}\right): \delta 160.6,137.3$, 133.6, 130.5, 128.2, 126.8, 124.2, 120.5, 110.6, 82.9, 46.7, 46.1, 24.7; HRMS (EI+): Calcd for $\mathrm{C}_{16} \mathrm{H}_{15} \mathrm{ClO}\left(\mathrm{M}^{+}\right)$: 258.0806 , Found 258.0811; Optical Rotation: $[\alpha]_{\mathrm{D}}{ }^{20}+32.0$ ( $c$ 0.75 in $\mathrm{CHCl}_{3}$ ) for an enantiomerically enriched sample of 96:4 er. The enantiomeric purity was established by HPLC analysis using a chiral column (Lux 3u Cellulose-1 column, $22{ }^{\circ} \mathrm{C}, 0.3 \mathrm{~mL} / \mathrm{min}, 99: 1$ Hexane:Isopropanol, $254 \mathrm{~nm}, \mathrm{t}_{\text {minor }}=24.827 \mathrm{~min}, \mathrm{t}_{\text {major }}$ $=25.692 \mathrm{~min}$ ). Absolute stereochemistry was determined through analogy with S10. See supporting information part B for HPLC chromatograms. 


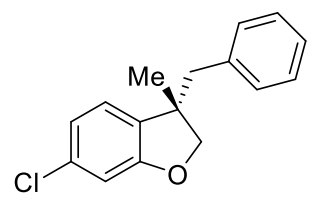

Synthesis of CB2 Receptor Agonist 23:

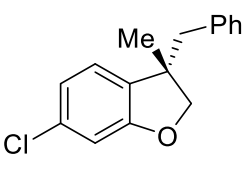

22

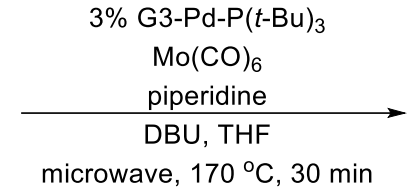

$57 \%$ yield

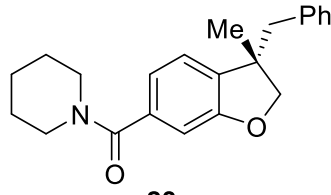

23

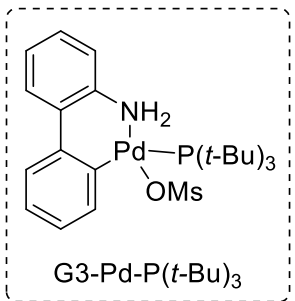

A modified literature procedure was followed. ${ }^{14}$ To a microwave vial $(2-5 \mathrm{~mL}$, Biotage Code No.: 351521$)$ with a Teflon coated stir bar was added G3-Pd-P $(t-\mathrm{Bu})_{3}(0.003 \mathrm{mmol}$, $3 \mathrm{~mol} \%)^{15}$, aryl chloride 22 ( $0.10 \mathrm{mmol}, 1.0$ equiv), $\mathrm{Mo}(\mathrm{CO})_{6}(0.10 \mathrm{mmol}, 1.0$ equiv), piperidine $(0.50 \mathrm{mmol}, 5.0$ equiv) and THF $(1.0 \mathrm{~mL})$, sequentially. DBU $(0.30 \mathrm{mmol}$, 3.0 equiv) was thereafter added, followed by rapid sealing of the vial under air. The vial was directly heated at $170{ }^{\circ} \mathrm{C}$ by microwave irradiation for $30 \mathrm{~min}$. After cooling to room temperature, the black reaction mixture was quenched upon addition of $\mathrm{H}_{2} \mathrm{O}(2 \mathrm{~mL})$ and was extracted with EtOAc $(3 \times 5 \mathrm{~mL})$. The combined organic layers were dried over $\mathrm{MgSO}_{4}$, filtered, and concentrated. The residue was purified by silica gel column chromatography ( $\mathrm{Hex}: \mathrm{Et}_{2} \mathrm{O}=1: 3$ ) to obtain the desired product 23 as white solid in $57 \%$ yield (19.1 mg). ${ }^{16}{ }^{1} \mathbf{H}$ NMR (400 MHz, $\mathbf{C D C l}_{\mathbf{3}}$ ): $\delta$ 7.25-7.20 (app. br s, 3H), 7.06-6.97 $(\mathrm{m}, 2 \mathrm{H}), 6.94(\mathrm{~d}, J=7.6 \mathrm{~Hz}, 1 \mathrm{H}), 6.89(\mathrm{~d}, J=7.6 \mathrm{~Hz}, 1 \mathrm{H}), 6.75(\mathrm{~s}, 1 \mathrm{H}), 4.53$ (d, $J=8.7$ Hz, 1H), 4.09 (d, $J=8.7 \mathrm{~Hz}, 1 \mathrm{H}), 3.69$ (app. br s, 2H), 3.34 (app. br s, 2H), 2.90 (d, $J=$ $13.5 \mathrm{~Hz}, 1 \mathrm{H}$ ), 2.86 (d, $J=13.5 \mathrm{~Hz}, 1 \mathrm{H}$ ), 1.67 (app. br s, 3H), 1.52 (app. br s, 3H), 1.36 (s, $3 \mathrm{H}) ;{ }^{13} \mathbf{C}$ NMR (100 MHz, $\left.\mathbf{C D C l}_{3}\right): \delta 170.3,159.6,137.4,136.9,136.3,130.5,128.1$, 126.7, 123.5, 119.2, 108.3, 82.4, 48.9, 46.7, 46.3, 43.3, 26.7, 25.8, 24.8, 24.7; Optical Rotation: $[\alpha]_{\mathrm{D}}{ }^{20}+12.2\left(c 0.75\right.$ in $\left.\mathrm{CHCl}_{3}\right)$ for an enantiomerically enriched sample of 96:4 er.

Determination of Absolute Configuration (Synthesis of Derivative S10):

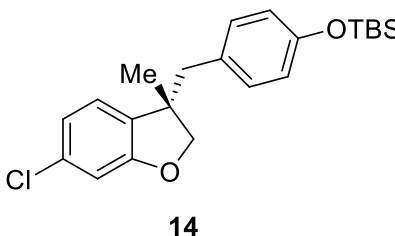

14

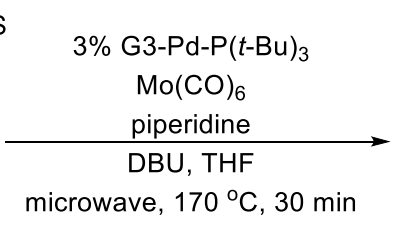

microwave, $170^{\circ} \mathrm{C}, 30 \mathrm{~min}$

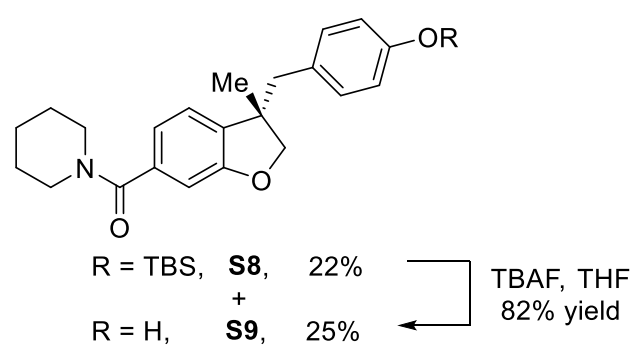


A similar procedure was followed as above. To a microwave vial $(2-5 \mathrm{~mL}$, Biotage Code No.: 351521$)$ with a Teflon coated stir bar was added G3-Pd-P $(t-\mathrm{Bu})_{3}(0.0015 \mathrm{mmol}, 3$ mol \%), aryl chloride 14 (0.050 mmol, 1.0 equiv), $\mathrm{Mo}(\mathrm{CO})_{6}(0.050 \mathrm{mmol}, 1.0$ equiv), piperidine $(0.25 \mathrm{mmol}, 5.0$ equiv) and THF $(0.50 \mathrm{~mL})$, sequentially. DBU $(0.15 \mathrm{mmol}$, 3.0 equiv) was thereafter added, followed by rapid sealing of the vial under air. The vial was directly heated at $170{ }^{\circ} \mathrm{C}$ by microwave irradiation for $30 \mathrm{~min}$. After cooling to room temperature, the black reaction mixture was quenched upon addition of $\mathrm{H}_{2} \mathrm{O}(2 \mathrm{~mL})$ and was extracted with EtOAc $(3 \times 3 \mathrm{~mL})$. The combined organic layers were dried over $\mathrm{MgSO}_{4}$, filtered, and concentrated. The residue was purified by silica gel column chromatography to obtain a mixture of silyl ether product $\mathbf{S 8}$ (colorless oil, 22\% yield, gradient wash till $\mathrm{Hex}: \mathrm{Et}_{2} \mathrm{O}=1: 3$ ) and deprotected phenol product $\mathbf{S 9}$ (white solid, 25\% yield, gradient wash till $\left.\mathrm{Hex}: \mathrm{Et}_{2} \mathrm{O}=1: 5\right)$.

Silyl ether product $\mathbf{S 8}$ was then subjected to deprotection conditions. ${ }^{12}$ A solution of $\mathbf{S 8}$ (0.011 mmol, 1.0 equiv) in dry THF $(0.50 \mathrm{~mL})$ was treated with tetrabutylammonium fluoride (1.0 M solution in THF, $0.033 \mathrm{mmol}, 3.0$ equiv), and was allowed to stir at room temperature for $3 \mathrm{~h}$. The reaction was quenched with $\mathrm{H}_{2} \mathrm{O}(1 \mathrm{~mL})$ and brine $(1 \mathrm{~mL})$ and the mixture was extracted with EtOAc $(3 \times 2 \mathrm{~mL})$. The combined organic layers were dried over $\mathrm{MgSO}_{4}$, filtered, and concentrated. The residue was purified by silica gel column chromatography $\left(\mathrm{Hex}: \mathrm{Et}_{2} \mathrm{O}=1: 5\right)$ to obtain the desired product $\mathbf{S 9}$ in $82 \%$ yield. Then the two portions of $\mathbf{S 9}$ were combined for further reaction.

${ }^{1} \mathbf{H}$ NMR of S8 (400 MHz, $\left.\mathbf{C D C l}_{3}\right): \delta 6.90-6.82(\mathrm{~m}, 4 \mathrm{H}), 6.75(\mathrm{~d}, J=1.2 \mathrm{~Hz}, 1 \mathrm{H}), 6.73-$ $6.68(\mathrm{~m}, 2 \mathrm{H}), 4.50(\mathrm{~d}, J=8.7 \mathrm{~Hz}, 1 \mathrm{H}), 4.08(\mathrm{~d}, J=8.7 \mathrm{~Hz}, 1 \mathrm{H}), 3.68$ (app. br s, 2H), 3.34 (app. br s, 2H), $2.82(\mathrm{~d}, J=13.5 \mathrm{~Hz}, 1 \mathrm{H}), 2.78(\mathrm{~d}, J=13.5 \mathrm{~Hz}, 1 \mathrm{H}), 1.72-1.52$ (br m, 6H), 1.33 (s, 3H), 0.97 (s, 9H), $0.18(\mathrm{~s}, 6 \mathrm{H})$.

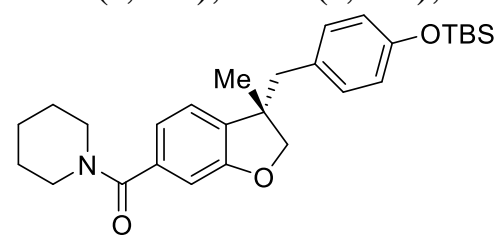

${ }^{1} \mathbf{H}$ NMR of S9 (400 MHz, $\left.\mathbf{C D C l}_{3}\right): \delta 6.92-6.86(\mathrm{~m}, 2 \mathrm{H}), 6.82(\mathrm{~d}, J=8.0 \mathrm{~Hz}, 2 \mathrm{H}), 6.74$ $(\mathrm{s}, 1 \mathrm{H}), 6.67$ (d, $J=8.0 \mathrm{~Hz}, 2 \mathrm{H}), 5.37$ (br s, 1H), 4.49 (d, $J=8.7 \mathrm{~Hz}, 1 \mathrm{H}), 4.09$ (d, $J=8.7$ $\mathrm{Hz}, 1 \mathrm{H}), 3.69$ (app. br s, 2H), 3.34 (app. br s, 2H), 2.79 (d, $J=14.2 \mathrm{~Hz}, 1 \mathrm{H}), 2.76$ (d, $J=$ $14.2 \mathrm{~Hz}, 1 \mathrm{H}), 1.73-1.48$ (br m, 6H), 1.34 (s, 3H).

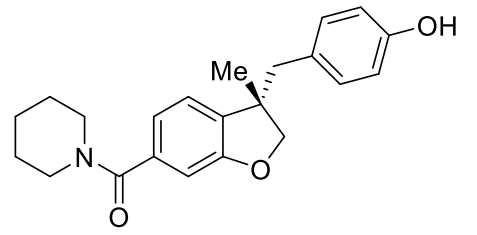




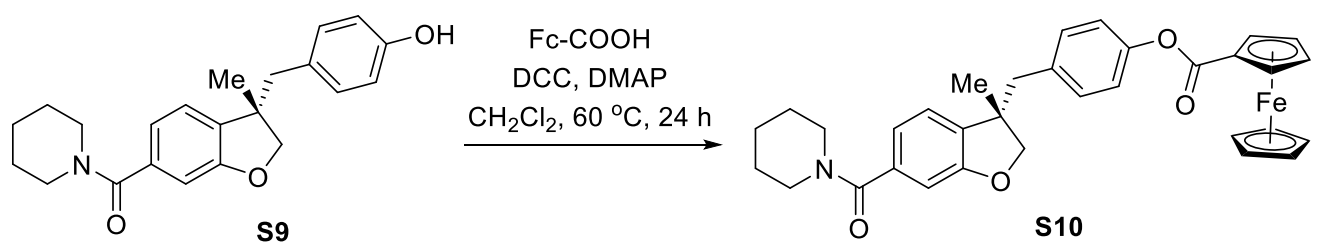

A modified literature procedure was followed. ${ }^{17}$ To a $\sim 10 \mathrm{~mL}$ screw-capped vial $(13 \mathrm{x}$ $100 \mathrm{~mm}$ ) was added phenol derivative $\mathbf{S 9}(7.0 \mathrm{mg}, 0.020 \mathrm{mmol}, 1.0$ equiv), ferrocenecarboxylic acid ( $0.021 \mathrm{mmol}, 1.05$ equiv), DMAP $(0.020 \mathrm{mmol}, 1.0$ equiv) and DCC $(0.030 \mathrm{mmol}, 1.5 \mathrm{eq})$. The vial was then evacuated and refilled with $\mathrm{N}_{2}$, followed by addition of $0.50 \mathrm{~mL} \mathrm{CH}_{2} \mathrm{Cl}_{2}$. After sealing the vial with a Teflon lined screw cap, the mixture was allowed to stir at $60{ }^{\circ} \mathrm{C}$ for $24 \mathrm{~h}$. The reaction was quenched upon addition of $\mathrm{H}_{2} \mathrm{O}(2 \mathrm{~mL})$ and the mixture was extracted with $\mathrm{CH}_{2} \mathrm{Cl}_{2}(3 \times 2 \mathrm{~mL})$. The combined organic layers were dried over $\mathrm{MgSO}_{4}$, filtered, and concentrated. The residue was purified by silica gel column chromatography $\left(\mathrm{Hex}: \mathrm{Et}_{2} \mathrm{O}=1: 5\right)$ to obtain the desired product $\mathbf{S 1 0}$ as orange solid ( $4.8 \mathrm{mg}, 43 \%$ yield). Single crystal of $\mathbf{S 1 0}$ was obtained by slow evaporation of an $\mathrm{Et}_{2} \mathrm{O} /$ heptane solution. ${ }^{1} \mathbf{H}$ NMR $\left(\mathbf{6 0 0} \mathbf{~ M H z}, \mathbf{C D C l}_{3}\right): \delta 7.10-7.00$ $(\mathrm{m}, 4 \mathrm{H}), 6.95(\mathrm{~d}, J=7.6 \mathrm{~Hz}, 1 \mathrm{H}), 6.90(\mathrm{dd}, J=7.4,1.5 \mathrm{~Hz}, 1 \mathrm{H}), 6.78(\mathrm{~d}, J=1.4 \mathrm{~Hz}, 1 \mathrm{H})$, 4.95 (app. br s, 2H), 4.54 (d, $J=8.8 \mathrm{~Hz}, 1 \mathrm{H}), 4.51-4.48$ (app. br s, 2H), 4.30 (s, 5H), 4.12 (d, $J=8.6 \mathrm{~Hz}, 1 \mathrm{H}), 3.69$ (app. br s, 2H), 3.35 (app. br s, $2 \mathrm{H}), 2.92$ (d, $J=13.4 \mathrm{~Hz}, 1 \mathrm{H}$ ), $2.89(\mathrm{~d}, J=13.7 \mathrm{~Hz}, 1 \mathrm{H}), 1.70-1.56(\mathrm{br} \mathrm{m}, 6 \mathrm{H}), 1.38(\mathrm{~s}, 3 \mathrm{H})$. Structure was unambiguously determined by $\mathrm{X}$-ray diffraction. 
— Preparation of Intermediates for Enantioselective Diarylation of Alkenes:

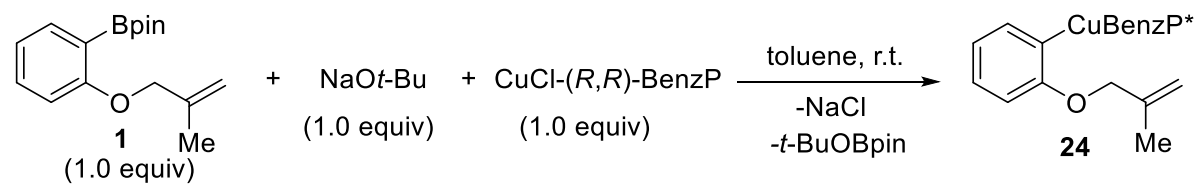

In an $\mathrm{N}_{2}$ filled glovebox, a $\sim 6 \mathrm{~mL}$ screw-capped vial $(17 \mathrm{x} 60 \mathrm{~mm})$ was charged with $\mathrm{CuCl}-(R, R)$-BenzP $(0.10 \mathrm{mmol}, 38.1 \mathrm{mg}), \mathrm{NaO} t$-Bu $(99.9 \%$ purity from Aldrich) (0.1 mmol, $9.6 \mathrm{mg})$, ArBpin $1(0.10 \mathrm{mmol}, 27.4 \mathrm{mg})$ and toluene $(1.0 \mathrm{~mL})$. The vial was sealed with a screw cap and allowed to stir at room temperature for $4 \mathrm{~h}$. The yellow slurry was filtered through Celite. Toluene was then removed under vacuum and cold pentane was added. The mixture was stored in a $-30{ }^{\circ} \mathrm{C}$ freezer overnight. The liquid portion of the mixture was then removed by pipette and the solid residue was dried under vacuum to obtain 24 as yellow solid (37.0 $\mathrm{mg}, 0.075 \mathrm{mmol}, 75 \%$ yield). ${ }^{1} \mathrm{H}$ NMR and ${ }^{31} \mathrm{P}$ NMR were then recorded at $25{ }^{\circ} \mathrm{C}$.

ArCuBenzP* (24): ${ }^{1} \mathbf{H}$ NMR (400 MHz, toluene-d $)$ : $\delta 8.05(\mathrm{dd}, J=6.3,2.2 \mathrm{~Hz}, 1 \mathrm{H})$, 7.37-7.29 (br m, 2H), 7.28-7.18 (m, 2H), 7.06 (dd, $J=5.8,3.4 \mathrm{~Hz}, 2 \mathrm{H}), 6.94$ (dd, $J=7.6$, $1.5 \mathrm{~Hz}, 1 \mathrm{H}), 5.22(\mathrm{~s}, 1 \mathrm{H}), 4.87(\mathrm{~s}, 1 \mathrm{H}), 4.57-4.48(\mathrm{~m}, 2 \mathrm{H}), 1.81(\mathrm{~s}, 3 \mathrm{H}), 1.23(\mathrm{~s}, 6 \mathrm{H}), 0.91$ (app. t, $J=7.2 \mathrm{~Hz}, 18 \mathrm{H}) ;{ }^{31}$ P NMR (170 MHz, toluene- $\left.\boldsymbol{d}_{\mathbf{8}}\right): \delta-7.8$.

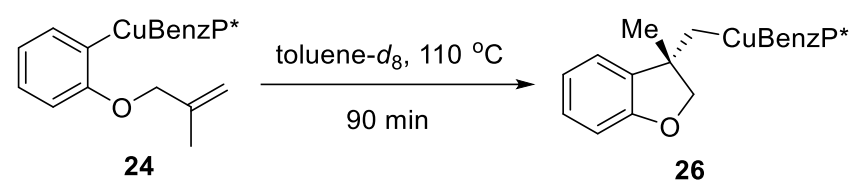

Complex 24 (2 mg) was heated in a gas tight NMR tube to $110{ }^{\circ} \mathrm{C}$ for 90 minutes in toluene- $d_{8}(0.5 \mathrm{~mL})$. The yellow solution turned dark yellow. ${ }^{1} \mathrm{H}$ NMR and ${ }^{31} \mathrm{P} \mathrm{NMR}$ were then recorded at $25{ }^{\circ} \mathrm{C}$.

Alkyl-CuBenzP* (26): ${ }^{1}$ H NMR (400 MHz, toluene-d s): $\delta 7.47(\mathrm{dd}, J=7.2,1.5 \mathrm{~Hz}$, 1H), 7.32-7.24 (br m, 2H), 7.07-7.01 (m, 2H), 6.94-6.87 (m, 2H), 6.80 (d, J=8.6 Hz, 1H), $4.74(\mathrm{~d}, J=7.4 \mathrm{~Hz}, 1 \mathrm{H}), 4.55(\mathrm{~d}, J=7.4 \mathrm{~Hz}, 1 \mathrm{H}), 1.77(\mathrm{~s}, 3 \mathrm{H}), 1.75(\mathrm{~d}, J=12.5 \mathrm{~Hz}, 1 \mathrm{H})$, $1.43(\mathrm{~d}, J=12.5 \mathrm{~Hz}, 1 \mathrm{H}), 1.11$ (s, 6H), 0.80 (app. $\mathrm{t}, J=7.1 \mathrm{~Hz}, 18 \mathrm{H}) ;{ }^{31} \mathbf{P}$ NMR (170 MHz, toluene- $\left.d_{8}\right): \delta-9.2$. 
To further confirm the identity of complexes $\mathbf{2 4}$ and $\mathbf{2 6}$, several experiments were carried out:

(1) Using 19 as reaction catalyst

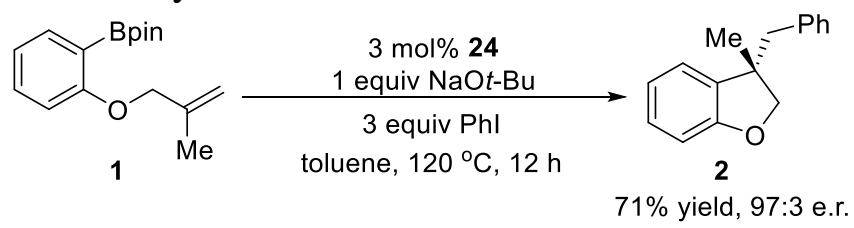

In an $\mathrm{N}_{2}$ filled glovebox, to a $\sim 10 \mathrm{~mL}$ screw-capped vial $(13 \times 100 \mathrm{~mm})$ was added the above isolated ArCuBenzP* 19 (0.003 mmol, $3 \mathrm{~mol} \%$ ), $\mathrm{NaOt}$-Bu (0.1 mmol, 1.0 equiv), ArBpin 1 ( $0.1 \mathrm{mmol}, 1.0$ equiv), iodobenzene ( $0.3 \mathrm{mmol}, 3.0$ equiv) and toluene (3.0 $\mathrm{mL})$. The vial was sealed with a Teflon lined screw cap and then removed from the glovebox. The mixture was allowed to stir at $120{ }^{\circ} \mathrm{C}$ for $12 \mathrm{~h}$. After cooling down to room temperature, the reaction was quenched upon addition of $\mathrm{H}_{2} \mathrm{O}(2 \mathrm{~mL})$ and extracted with EtOAc $(3 \times 2 \mathrm{~mL})$. The combined organic layers were dried over $\mathrm{MgSO}_{4}$, filtered, and concentrated. The residue was purified by silica gel column chromatography (Hex : $\mathrm{DCM}=15: 1)$ to obtain the desired product 2 in $71 \%$ isolated yield $(15.9 \mathrm{mg}, 0.071 \mathrm{mmol})$ and $97: 3$ e.r..

(2) Addition of PhI to 24

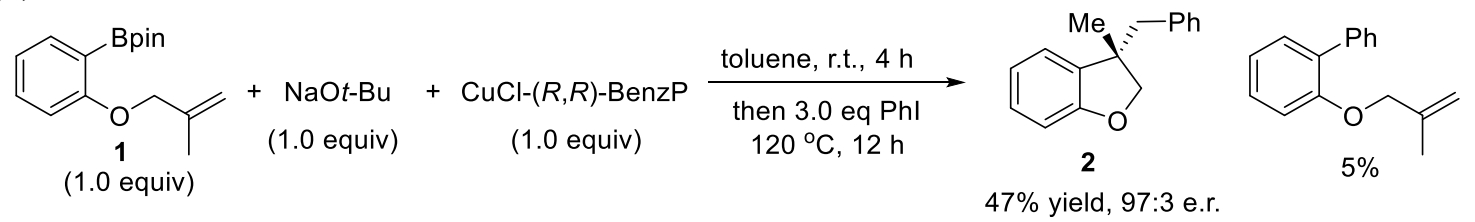

In an $\mathrm{N}_{2}$ filled glovebox, a $\sim 6 \mathrm{~mL}$ screw-capped vial $(17 \times 60 \mathrm{~mm})$ was charged with CuCl-(R,R)-BenzP (0.020 mmol, $7.6 \mathrm{mg}), \mathrm{NaO} t$-Bu $(0.020 \mathrm{mmol}, 1.9 \mathrm{mg})$, ArBpin 1 $(0.020 \mathrm{mmol}, 5.5 \mathrm{mg})$ and toluene $(2.0 \mathrm{~mL})$. The vial was allowed to stir at room temperature for $4 \mathrm{~h}$, followed by addition of iodobenzene $(0.060 \mathrm{mmol}, 3.0$ equiv). The vial was sealed with the Teflon lined screw cap and then removed from the glovebox. The mixture was allowed to stir at $120{ }^{\circ} \mathrm{C}$ for $12 \mathrm{~h}$. After cooling down to room temperature, the reaction was quenched upon addition of $\mathrm{H}_{2} \mathrm{O}(2 \mathrm{~mL})$ and extracted with EtOAc ( $3 \times 2 \mathrm{~mL})$. The combined organic layers were dried over $\mathrm{MgSO}_{4}$, filtered, and concentrated. The residue was purified by silica gel column chromatography (Hex : $\mathrm{DCM}=15: 1)$ to obtain the desired product 2 in $47 \%$ isolated yield $(2.1 \mathrm{mg}, 0.0094 \mathrm{mmol})$ and $97: 3$ e.r..

(3) Addition of PhI to 26

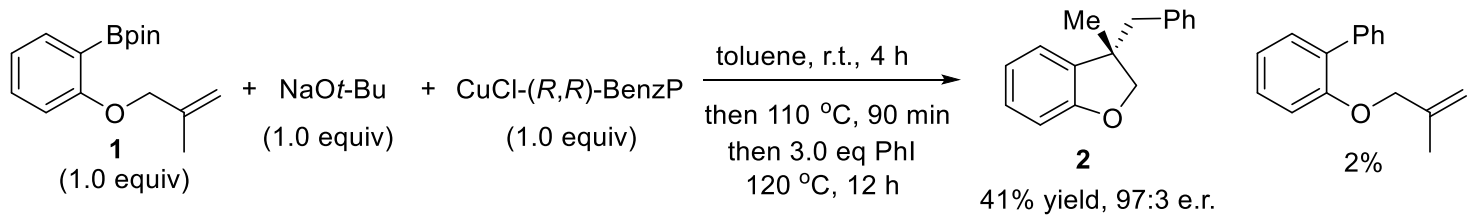


In an $\mathrm{N}_{2}$ filled glovebox, a $\sim 6 \mathrm{~mL}$ screw-capped vial $(17 \mathrm{x} 60 \mathrm{~mm})$ was charged with $\mathrm{CuCl}-(R, R)$-BenzP $(0.020 \mathrm{mmol}, 7.6 \mathrm{mg}), \mathrm{NaO} t$-Bu $(0.020 \mathrm{mmol}, 1.9 \mathrm{mg})$, ArBpin 1 $(0.020 \mathrm{mmol}, 5.5 \mathrm{mg})$ and toluene $(2.0 \mathrm{~mL})$. The vial was sealed with a Teflon lined screw cap and allowed to stir at room temperature for $4 \mathrm{~h}$. Then the vial was removed from the glovebox. The yellow mixture was allowed to stir at $110{ }^{\circ} \mathrm{C}$ for 90 minutes. After cooling down to ambient temperature, the solution was transferred into the glovebox, followed by addition of iodobenzene $(0.060 \mathrm{mmol}, 3.0$ equiv $)$. The vial was sealed with the Teflon lined screw cap and then removed from the glovebox. The mixture was allowed to stir at $120{ }^{\circ} \mathrm{C}$ for $12 \mathrm{~h}$. After cooling down to room temperature, the reaction was quenched upon addition of $\mathrm{H}_{2} \mathrm{O}(2 \mathrm{~mL})$ and extracted with EtOAc $(3 \times 2$ $\mathrm{mL}$ ). The combined organic layers were dried over $\mathrm{MgSO}_{4}$, filtered, and concentrated. The residue was purified by silica gel column chromatography (Hex : DCM $=15: 1$ ) to obtain the desired product 2 in $41 \%$ isolated yield $(1.8 \mathrm{mg}, 0.0082 \mathrm{mmol}$ ) and 97:3 e.r..

(1) Hatakeyama, T.; Hashimoto, T.; Kondo, Y.; Fujiwara, Y.; Seike, H.; Takaya, H.; Tamada, Y.; Ono, T.; Nakamura, M. J. Am. Chem. Soc. 2010, 132, 10674-10676.

(2) Tang, C.-Y.; Tao, Y.; Wu, X.-Y.; Sha, F. Adv. Synth. Catal. 2014, 356, 609-615.

(3) Aslanidis, P.; Cox, P. J.; Kaltzoglou, A.; Tsipis, A. C. Eur. J. Inorg. Chem. 2006, 334-344.

(4) You, W.; Brown, M. K. J. Am. Chem. Soc. 2014, 136, 14730-14733.

(5) 2-Bromo-5-methoxyphenol was prepared according to literature procedure: Kitching, M. O.; Hurst, T. E.; Snieckus, V. Angew. Chem., Int. Ed. 2012, 51, 2925-2929. And it reacted with 3-chloro-2-methyl-1propene according to literature procedure: Rueping, M.; Leiendecker, M.; Das, A.; Poisson, T.; Bui, L. Chem. Commun. 2011, 47, 10629-10631.

(6) 5-Chloro-2-iodophenol was prepared according to literature procedure: Lee, E.; Kamlet, A. S.; Powers, D. C.; Neumann, C. N.; Boursalian, G. B.; Furuya, T.; Choi, D. C.; Hooker, J. M.; Ritter, T. Science 2011, 334, 639-642. And it reacted with 3-chloro-2-methyl-1-propene according to literature procedure: Rueping, M.; Leiendecker, M.; Das, A.; Poisson, T.; Bui, L. Chem. Commun. 2011, 47, 10629-10631.

(7) Li, W.; Nelson, D. P.; Jensen, M. S.; Hoerrner, R. S.; Cai, D.; Larsen, R. D.; Reider, P. J. J. Org. Chem. 2002, 67, 5394-5397.

(8) 1-((2-(Chloromethyl)allyl)oxy)-2-iodobenzene was prepared according to literature procedure: Newman, S. G.; Howell, J. K.; Nicolaus, N.; Lautens M. J. Am. Chem. Soc. 2011, 133, 14916-14919. And it reacted with (2-methylallyl)zinc(II) chloride solution ( $0.88 \mathrm{M}$ in THF, prepared according to literature procedure: Han, C.; Buchwald, S. L. J. Am. Chem. Soc. 2009, 131, 7532-7533.) in THF at $50{ }^{\circ} \mathrm{C}$ overnight.

(9) 2-Methylenehexan-1-ol was prepared according to literature procedure: Ragoussis, V.; Giannikopoulos, A.; Skoka, E.; Grivas, P. J. Agric. Food Chem. 2007, 55, 5050-5052. And it reacted with 2-iodophenol according to literature procedure: Wipf, P.; Rodriguez, S. Adv. Synth. Catal. 2002, 344, 434-440.

(10) 3-Methyl-2-methylenebutan-1-ol was prepared according to literature procedure: Lee, J.; Parker, K. A. Org. Lett. 2012, 14, 2682-2685. And it reacted with 2-iodophenol according to literature procedure: Wipf, P.; Rodriguez, S. Adv. Synth. Catal. 2002, 344, 434-440.

(11) 3-Chloro-2-methyl-1-propene reacted with $N$-benzyl-2-iodoaniline according to literature procedure: Yang, H.; Sun, P.; Zhu, Y.; Yan, H.; Lu, L.; Liu, D.; Rong, G.; Mao, J. Catal. Commun. 2013, 38, 21-25.

(12) Kumar, G. D. K.; Natarajan, A. Tetrahedron Lett. 2008, 49, 2103-2105.

(13) Gil, G. S.; Groth U. M. J. Am. Chem. Soc. 2000, 122, 6789-6790.

(14) Lagerlund, O.; Larhed, M. J. Comb. Chem. 2006, 8, 4-6.

(15) Prepared according to literature procedure: Bruno, N. C.; Tudge, M. T.; Buchwald, S. L. Chem. Sci. 2013, 4, 916-920.

(16) Diaz, P.; Phatak, S. S.; Xu, J.; Fronczek, F. R.; Astruc-Diaz, F.; Thompson, C. M.; Cavasotto, C. N.; Naguib, M. ChemMedChem 2009, 4, 1615-1629.

(17) Bringmann, G.; Hinrichs, J.; Peters, K.; Peters, E.-M. J. Org. Chem. 2001, 66, 629-632. 